\title{
Nonlinear predictors for systems with bounded trajectories and delayed measurements
}

\author{
Stefano Battilotti ${ }^{\text {a }}$ \\ a Dipartimento di Ingegneria Informatica, Automatica e Gestionale "Antonio Ruberti” (e-mail: battilotti@dis.uniromH1.it).
}

\begin{abstract}
Novel nonlinear predictors are studied for nonlinear systems with delayed measurements without assuming globally Lipschitz conditions or a known predictor map but requiring instead bounded state trajectories. The delay is constant and known. These nonlinear predictors consists of a series of dynamic filters that generate estimates of the state vector (and its maximum magnitude) at different delayed time instants which differ from one another by a small fraction of the overall delay.
\end{abstract}

\section{Introduction}

The problem of reconstructing the unmeasurable state variables by using the system model and the available online output measurements has been addressed by many authors. Particularly challenging remains the nonlinear observer design problem in the presence of delayed output measurements. In this case it is important to implement some kind of prediction based on the delayed measurements. A nonlinear observer has been proposed in Marquez et al. (2000) for linearizable by additive output injection systems. A predictor based on a cascade of observers has been introduced with LMI techniques in Besancon et al. (2007). For globally Lipschitz continuous invertible observability maps (Germani et al. (2011)) the proposed observer consists of a chain of dynamic predictors that reconstruct the unmeasurable state vector at different delayed time-instants within the time-delay window introduced by the output measurements. Hence, the proposed nonlinear observer exhibits a chained structure that explicitly takes into account the magnitude of the output delay. The paper Kazantzis et al. (2013), while adopting a conceptually similar design methodology, aims at overcoming some of the restrictions associated with the above approaches by following a technically different path. Also globally Lipschitz conditions on the system are required in Ibrir (2011). In all these papers linear predictors are used. A survey on observers with measurement delay is found in Richard (2003) while a predictor-based approach is extensively surveyed in Krstic (2009).

Predictor-based results have been recently obtained in

\footnotetext{
^ This work was supported by MIUR.
}

Karafyllis et al. (2013) where a known compact absorbing set (plus some technical facts) is assumed for all the system trajectories. This assumption is much stronger than boundedness of the state trajectories, where the absorbing compact set depends on the initial condition of each state trajectory. On the other hand, these dynamic predictors follow the structure of the ones introduced in Germani et al. (2011) and Kazantzis et al. (2013).

Predictors, which are not implemented as dynamical filters, are designed in Karafyllis et al. (2012b) under the assumption that either a) the expression of the state trajectories is explicitly known or b) the system is globally Lipschitz. In Karafyllis et al. (2012a) the existence of predictor-based observers is shown under the hypothesis that the so-called predictor map is known exactly. Actually, all the above cited results can be implemented only if the predictor map is available (this happens for linear systems, bilinear systems, chains of linear systems with input nonlinearities), except for Karafyllis et al. (2013) where a modified version of the chained predictors, introduced in Germani et al. (2011) and Kazantzis et al. (2013), are used. Further results have been obtained for delays that depend on the delayed states in Bekiaris-Liberis et al. (2013). Numerical and approximate predictors have been proposed in Karafyllis et al. (2013). Design of predictors for specific implementation has been proposed in Mazenc et al. (2011).

In this paper we consider the problem of state observation for a class of systems which satisfy an incremental homogeneity (in the generalized sense) condition with bounded state trajectories. This class of systems includes lower triangular and upper triangular systems and many non-triangular systems. The measurement delay is con- 


\section{CONFIDENTIAL. Limited circulation. For review only Automatica submission 13-0695.3}

stant and known. It is not required any globally Lipschitz condition on the system or availability of the predictor map. An estimation of the maximum delayed state and its maximum magnitude is dynamically implemented and, using these estimates, a prediction is generated by a chain of nonlinear dynamic predictors that reconstruct the unmeasurable state vector at different delayed timeinstants. The novelty of our observers, on account of the strong nonlinearities of the system, is the use for the first time of nonlinear predictors with saturated estimates where the saturation level is tuned on-line according to the delayed measurements. This tuning is needed to estimate the width of the compact absorbing set of each state trajectory (depending on its initial condition). Our result is based on the observer design with undelayed measurements proposed in Battilotti (2011). A constructive design is illustrated by a step-by-step procedure.

\section{Notation}

(N1) $\mathbb{R}^{n}$ (resp. $\mathbb{R}^{n \times n}$ ) is the set of $n$-dimensional real column vectors (resp. $n \times n$ matrices). $\mathbb{R}_{\geqslant}$(resp. $\mathbb{R}_{\geqslant}^{n}$, $\mathbb{R}_{\geqslant}^{n \times n}$ ) denotes the set of real non-negative numbers (resp. vectors in $\mathbb{R}^{n}$, matrices in $\mathbb{R}^{n \times n}$, with real nonnegative entries). $\mathbb{R}_{>}$(resp. $\mathbb{R}_{>}^{n}$ ) denotes the set of real positive numbers (resp. vectors in $\mathbb{R}^{n}$ with real positive entries).

(N3) For any matrix $V \in \mathbb{R}^{p \times n}$ we denote by $V_{i j}$ the $(i, j)$-th entry of $V$ and for any vector $v \in \mathbb{R}^{n}$ we denote by $v_{i}$ the $i$-th element of $v$. We retain a similar notation for functions. For any $v \in \mathbb{R}^{n}$ we denote by $\operatorname{diag}\{v\}$ the diagonal $n \times n$ matrix with diagonal elements $v_{1}, \ldots, v_{n}$. Also, $|a|$ denotes the absolute value of $a \in \mathbb{R},\|a\|$ denotes the euclidean norm of $a \in \mathbb{R}^{n}$, $\|A\|$ denotes the norm of $A \in \mathbb{R}^{n \times n}$ induced from the euclidean norm $\|a\|$ and $\langle\langle a\rangle$ the column vector of the absolute values of the elements of $a \in \mathbb{R}^{n}$, i.e. $\left(\left|a_{1}\right| \cdots\left|a_{n}\right|\right)^{T}$.

(N3) We denote by $C^{j}(\mathscr{X}, \mathscr{Y})$, with $j \geqslant 0, \mathscr{X} \subset \mathbb{R}^{n}$ and $\mathscr{Y} \subset \mathbb{R}^{p}$, the set of $j$-times continuously differentiable functions $f: \mathscr{X} \rightarrow \mathscr{Y}$, by $L^{\infty}\left(\mathbb{R}_{\geqslant}, \mathscr{Y}\right)$ the set of functions $f \in C^{0}\left(\mathbb{R}_{\geqslant}, \mathscr{Y}\right)$ such that $\sup _{\theta \geqslant 0}\|f(\theta)\|<$ $+\infty$ and by $L^{j}\left(\mathbb{R}_{\geqslant}, \mathscr{Y}\right)$, with $j>0$, the set of $f \in$ $C^{0}\left(\mathbb{R}_{\geqslant}, \mathscr{Y}\right)$ such that $\int_{0}^{\infty}\|f(\theta)\|^{j} d \theta<+\infty$.

(N4) $D^{j}(\mathscr{X}, \mathscr{Z}), \mathscr{X}, \mathscr{Z} \subset \mathbb{R}^{n}$, is the set of functions $f \in C^{j}(\mathscr{X}, \mathscr{Z})$ with decoupled components, viz. $f(x)=\left(f_{1}\left(x_{1}\right), \cdots, f_{n}\left(x_{n}\right)\right)^{T}$. A saturation function $\sigma(h, \cdot)$ with levels $h \in \mathbb{R}_{>}^{n}$ is a function $\sigma(h, \cdot) \in D^{0}\left(\mathbb{R}^{n}, \mathbb{R}^{n}\right)$ such that for each $i=1, \ldots, n$ and $x \in \mathbb{R}^{n}: \sigma_{i}\left(h, x_{i}\right)=x_{i}$ if $\left|x_{i}\right| \leqslant h_{i}$ and $\sigma_{i}\left(h, x_{i}\right)=h_{i}$ otherwise. In other words, $\sigma_{i}\left(h, x_{i}\right)$ saturates $x_{i}$ if it exceeds the range $\left[-h_{i}, h_{i}\right]$.

(N5) For any vectors $x \in \mathbb{R}^{n}, \mathfrak{r} \in \mathbb{R}_{>}^{n}$ and $\varepsilon \in \mathbb{R}_{>}$, we define

$\varepsilon^{\mathfrak{r}}:=\left(\varepsilon^{\mathfrak{r}_{1}}, \cdots, \varepsilon^{\mathfrak{r}_{n}}\right)^{T}, \varepsilon^{\mathfrak{r}} \diamond x:=\left(\varepsilon^{\mathfrak{r}_{1}} x_{1}, \cdots, \varepsilon^{\mathfrak{r}_{n}} x_{n}\right)^{T}$

viz. $\varepsilon^{\mathfrak{r}} \diamond x$ is the dilation of a vector $x$ with weights $\mathfrak{r}$.
Note that for any $x, y \in \mathbb{R}^{n}, \mathfrak{r}_{1}, \mathfrak{r}_{2} \in \mathbb{R}_{>}^{n}$ and $\varepsilon \in \mathbb{R}_{>}$

$$
\begin{aligned}
& \varepsilon^{\mathfrak{r}_{1}} \diamond \varepsilon^{\mathfrak{r}_{2}} \diamond x=\varepsilon^{\mathfrak{r}_{2}} \diamond \varepsilon^{\mathfrak{r}_{1}} \diamond x=\varepsilon^{\mathfrak{r}_{1}+\mathfrak{r}_{2}} \diamond x, \\
& \left(\varepsilon^{\mathfrak{r}_{1}} \diamond x\right)^{T}\left(\varepsilon^{\mathfrak{r}_{2}} \diamond y\right)=\left(\varepsilon^{\mathfrak{r}_{2}} \diamond x\right)^{T}\left(\varepsilon^{\mathfrak{r}_{1}} \diamond y\right) \\
& =\left(\varepsilon^{\mathfrak{r}_{1}+\mathfrak{r}_{2}} \diamond x\right)^{T} y=x^{T}\left(\varepsilon^{\mathfrak{r}_{1}+\mathfrak{r}_{2}} \diamond y\right)
\end{aligned}
$$

(N6) for any vectors $x, y \in \mathbb{R}^{n}$ we write $x \leq y$ if and only if $x_{i} \leqslant y_{i}$ for all $i=1, \ldots, n$. We retain the same notation for matrices $A, B \in \mathbb{R}^{n \times n}: A \preceq B$ if and only if $A_{i j} \leqslant B_{i j}$ for all $i, j=1, \ldots, n$. On the other hand $A \geqslant B$ (resp. $A>B$ ) for matrices $A, B \in \mathbb{R}^{n \times n}$ if and only if $A-B$ is positive semidefinite (resp. positive definite).

\section{Main assumptions}

Consider the system

$$
\begin{aligned}
& \dot{x}(t)=f(x(t)):=A x(t)+\phi(x(t)), x(-\Delta):=x_{0}, \\
& y(t)=h(x(t-\Delta)):=C x(t-\Delta)+\psi(x(t-\Delta)), t \geqslant 0
\end{aligned}
$$

for $t \geqslant 0$, where $\Delta>0$ is the constant (known) measurement delay, $x \in \mathbb{R}^{n}, y \in \mathbb{R}$, the measurement $y$ is a function of the state at time $t-\Delta$. The matrix $A$ is in Brunowski canonical form and $C=\left(\begin{array}{llll}1 & 0 & \cdots & 0\end{array}\right)$. Moreover, $\phi$ and $\psi$ are locally Lipschitz continuous with $\phi(0)=0$ and $\psi(0)=0$. The vector of initial conditions $x(-\Delta)$ is $x_{0}$. We will denote by $x\left(t, x_{0}\right)\left(\right.$ resp. $\left.y\left(t, x_{0}\right)\right)$ the state (resp. output) trajectory of (4) ensuing from $x_{0}$ at $t=-\Delta$ (resp. at $\left.t=0\right)$ and $x\left(t, x_{0}\right)$ is unique and defined over its maximum right extension interval (theorem 3.7 and proposition 3.10 of Smith (2011)). The problem is to give a prediction of the state at time $t$ using the delayed measurement. Our assumptions are the following ones (see the appendix for a short review of incremental homogeneity in the upper bound):

(H0) (incremental homogeneity) $C^{T} \psi$ and $A^{T} \phi$ are incrementally homogeneous in the upper bound (i.h.u.b.) with quadruple $\left(\mathfrak{r}, \mathfrak{r}-\mathfrak{g}, \mathfrak{g}, C^{T} \Psi\right)$ and, respectively, $\left(\mathfrak{r}, \mathfrak{r}-\mathfrak{g}, \mathfrak{g}, A^{T} \Phi\right)$ and $\left(I-A A^{T}\right) \phi$ is incrementally homogeneous in the upper bound (i.h.u.b.) with quadruple $\left(\mathfrak{r},\left(I-A A^{T}\right)(\mathfrak{r}+\mathfrak{g}), \mathfrak{g},\left(I-A A^{T}\right) \Phi\right)$, where $\Phi(0,0)=0, \Psi(0,0)=0$ and

$$
\begin{aligned}
& 2\left(\mathfrak{g}_{j}-\mathfrak{g}_{j-1}\right)+\mathfrak{g}_{j-1}+\mathfrak{r}_{j-1} \leqslant \mathfrak{r}_{j}-\mathfrak{g}_{j} \leqslant \mathfrak{g}_{j-1}+\mathfrak{r}_{j-1}, \\
& j=2, \ldots, n,
\end{aligned}
$$

(H1) (boundedness) for each $x_{0} \in \mathbb{R}^{n}$ there exist a compact set $\mathscr{C}_{x_{0}} \subset \mathbb{R}^{n}$ such that $x\left(t, x_{0}\right) \in \mathscr{C}_{x_{0}} \forall t \geqslant$ $-\Delta$,

(H2) (incremental observability) for any $x_{0}^{\prime}, x_{0}^{\prime \prime} \in \mathbb{R}^{n}$ : $y\left(t, x_{0}^{\prime}\right)=y\left(t, x_{0}^{\prime \prime}\right) \forall t \geqslant 0 \Rightarrow x\left(t, x_{0}^{\prime}\right)=x\left(t, x_{0}^{\prime \prime}\right) \forall t \geqslant$ $-\Delta$. 
Remark 1 Assumptions $\mathrm{HO}$ states that $C^{T} \psi, A^{T} \phi$ and $\left(I-A A^{T}\right) \phi$ are incrementally homogeneous with a certain relation between degrees and weights specified by (5). $\mathrm{HO}$ is a condition for the existence of a semi-global linear observer for the undelayed state $x(t-\Delta)$ (see Battilotti (2013)). It can be seen that assumption HO is satisfied for large classes of nonlinear systems:

(i) with locally Lipschitz lower triangular (or normbounded by lower triangular maps) $\phi$ :

$\phi(x):=\left(\phi_{1}\left(x_{1}\right), \cdots, \phi_{n}\left(x_{1}, x_{2}, \ldots, x_{n}\right)\right)^{T}$

and $\psi(x):=0$, where each $\phi_{j}, j=1, \ldots, n$, is (normbounded by) a sum of terms with the form $x_{j_{1}}^{t_{j_{1}}} \cdots x_{j_{l}}^{t_{j_{l}}}$ for some reals $t_{j_{i}} \geqslant 1$. For example in the case of $\phi(x):=\left(x_{1}^{\frac{3}{2}}, x_{1}^{2} x_{2}^{3}\right)^{T}$ we choose $\mathfrak{r}=\left(\frac{1}{2}, 1\right)^{T}, \mathfrak{g}=$ $(6,2)^{T}$.

(ii) with locally Lipschitz strict upper triangular (or norm-bounded by strict upper triangular maps) $\phi$ :

$\phi(x):=\left(\phi_{1}\left(x_{3}, \ldots, x_{n}\right), \cdots, \phi_{n-2}\left(x_{n}\right), 0,0\right)^{T}$

and $\psi(x):=\psi_{1}\left(x_{2}, \ldots, x_{n}\right)$, where each $\phi_{j}, j=$ $1, \ldots, n-2$, and $\psi$ is (norm-bounded by) a sum of terms with the form $x_{j_{1}}^{t_{j_{1}}} \cdots x_{j_{l}}^{t_{j_{l}}}$ for some reals $t_{j_{i}} \geqslant 1$. For example in the case of $\phi(x):=\left(x_{3} x_{4}, x_{4}^{2}, 0,0\right)^{T}$ and $\psi(x):=x_{2} x_{4}$ we choose $\mathfrak{r}=(8,6,4,1)^{T}$, $\mathfrak{g}=(-1,-1,-1,-2)^{T}$.

(iii) for locally Lipschitz homogeneous (in the classical sense) $\phi$ and $\psi$ with weights such that $\mathfrak{r}_{j+1}-\mathfrak{r}_{j}=2 \mathfrak{g}_{0}$ for all $j=1, \ldots, n-1$ and homogeneity degree $2 \mathfrak{g}_{0}$ and, resp., 0. For example $\phi(x):=\left(0, x_{2}^{p}\right)^{T}$ for $1<p<2$, where we choose $\mathfrak{r}=(2(2-p), 2)^{T}, \mathfrak{g}=(p-1, p-1)^{T}$.

Remark 2 H1 and H2 (Battilotti (2011)) are extra conditions to render global the semi-global observer of Battilotti (2013) by using saturated estimates with dynamically tuned saturation levels. Assumption H1 is somewhat restrictive. However, many physical systems have this property (Van Der Pol and Fitzhugh-Nagumo oscillators, Lorentz-like systems: see section 6 for examples). Note that we do not require the knowledge of a Lyapunov function for the system. $\square$

\section{The structure of the predictor}

Wherever possible we will omit the dependence of the state trajectories from the initial conditions. The following notation is adopted for the delayed state vectors and measurements:

$$
\begin{aligned}
x^{(j)}(t) & :=x\left(t-\Delta+j \frac{\Delta}{m}\right), \\
y^{(j)}(t) & :=y\left(t+j \frac{\Delta}{m}\right), j=0, \ldots, m .
\end{aligned}
$$

The predictor we propose has the following structure. A first block is devoted, on one hand, to the estimation $\xi^{(0)}(t)$ of $x^{(0)}(t), t \geqslant 0$,

$$
\begin{aligned}
& \dot{\xi}^{(\mathbf{0})}(\mathbf{t})=\mathbf{M}\left(\boldsymbol{\mu}^{(\mathbf{0})}(\mathbf{t}), \boldsymbol{\xi}^{(\mathbf{0})}(\mathbf{t})\right) \\
& +\mathbf{L}\left(\boldsymbol{\mu}^{(\mathbf{0})}(\mathbf{t})\right)\left[\mathbf{y}(\mathbf{t})-\mathbf{Y}\left(\boldsymbol{\mu}^{(\mathbf{0})}(\mathbf{t}), \boldsymbol{\xi}^{(\mathbf{0})}(\mathbf{t})\right)\right]
\end{aligned}
$$

where

$$
\begin{aligned}
& M(\mu, \xi):=A \xi+\phi\left(\sigma\left(c \mu^{\mathfrak{r}}, \xi\right)\right) \\
& L(\mu):=\left(I-A^{T} \mathfrak{G}(\mu)\right)^{-1} C^{T} K \mu^{2 C \mathfrak{g}}, \\
& \mathfrak{G}(\mu):=\operatorname{diag}\left\{\Gamma \mu^{2 A \mathfrak{g}}\right\}, Y(\mu, \xi):=C \xi+\psi\left(\sigma\left(c \mu^{\mathfrak{r}}, \xi\right)\right),(8)
\end{aligned}
$$

for some saturation function $\sigma$ with levels $c \mu^{\mathfrak{r}}$ (see (N4) in the notation section), $c, K>0$ and diagonal positive definite $\Gamma \in \mathbb{R}^{n \times n}$, and, on the other, to the estimation $\mu^{(0)}(t)$ of the maximum magnitude of $x^{(0)}(t)$ over $[0,+\infty)$

$\dot{\boldsymbol{\mu}}^{(\mathbf{0})}(\mathbf{t})=\mathbf{G}\left(\boldsymbol{\mu}^{(\mathbf{0})}(\mathbf{t}), \boldsymbol{\xi}^{(\mathbf{0})}(\mathbf{t})\right)+\mathbf{Q}\left(\boldsymbol{\mu}^{(\mathbf{0})}(\mathbf{t}), \boldsymbol{\xi}^{(\mathbf{0})}(\mathbf{t}), \mathbf{y}(\mathbf{t})\right)(9)$

where

$G(\mu, \xi):=\mu^{1-2\left|\min _{i} \mathfrak{g}_{i}\right|}\left\|\mu^{-\mathfrak{r}} \diamond \sigma\left(c \mu^{\mathfrak{r}}, \xi-\sigma\left(c \mu^{\mathfrak{r}}, \xi\right)\right)\right\|^{2}(10)$ $Q(\mu, \xi, y):=\mu^{1-2\left|\min _{i} \mathfrak{g}_{i}\right|} \| \mu^{-\mathfrak{r}} \diamond \sigma\left(c \mu^{\mathfrak{r}}, C^{T}\left(y-Y(\mu, \xi) \|^{2}\right.\right.$

(see (1) for definitions).

The estimator (7) is a copy of the system equations (4) plus a innovation term $L\left(\mu^{(0)}\right)\left[y-Y\left(\mu^{(0)}, \xi^{(0)}\right)\right]$ in which the gain matrix $L\left(\mu^{(0)}\right)$ depends adaptively on $\mu^{(0)}$ and the estimate $\xi^{(0)}$ is saturated with levels $c\left(\mu^{(0)}\right)^{\mathfrak{r}}$. The estimate $\mu^{(0)}(t)$ is positive and increasing in time and approaches a constant value which depends on (the unknown value of $x^{(0)}(0)$ and gives an estimate of the maximum magnitude of $x^{(0)}(t)$ over $[0,+\infty)$, which by assumption $H 1$ is bounded (but unknown depending on the initial condition $\left.x^{(0)}(0)\right)$. While $\mu^{(0)}(t)$ tends to this constant value, the estimate $\xi^{(0)}(t)$ tends to desaturate and the terms $Q(t)$ and $G(t)$ in (9) tend to zero. The saturation of the estimates allows to cope with large initial state estimation errors and drive these errors to a sufficiently small value for which the estimate $\xi^{(0)}(t)$ is no longer saturated. When the saturations are not active any more in (4)-(7)-(9), the estimation error $x^{(0)}(t)-\xi^{(0)}(t)$ tends to zero (by assumption H2).

The estimates $\xi^{(0)}$ and $\mu^{(0)}$ are used in the block devoted, on one hand, to the estimation $\xi^{(1)}(t)$ of $x^{(1)}(t), t \geqslant 0$ (one-step prediction),

$\dot{\xi}^{(1)}(\mathbf{t})=\dot{\xi}^{(0)}(\mathbf{t})$

$+\mathbf{M}\left(\boldsymbol{\mu}^{(\mathbf{1})}(\mathbf{t}), \xi^{(\mathbf{1})}(\mathbf{t})\right)-\mathbf{M}\left(\boldsymbol{\mu}^{(\mathbf{1})}\left(\mathbf{t}-\frac{\Delta}{\mathbf{m}}\right), \xi^{(\mathbf{1})}\left(\mathbf{t}-\frac{\Delta}{\mathbf{m}}\right)\right)$ 
and, on the other, to the estimation $\mu^{(1)}(t)$ of $v^{(1)}(t):=$ $\mu^{(0)}\left(t+\frac{\Delta}{m}\right), t \geqslant 0$,

$\dot{\boldsymbol{\mu}}^{(\mathbf{1})}(\mathbf{t})=\dot{\boldsymbol{\mu}}^{(\mathbf{0})}(\mathbf{t})$

$+\mathbf{G}\left(\boldsymbol{\mu}^{(\mathbf{1})}(\mathbf{t}), \boldsymbol{\xi}^{(\mathbf{1})}(\mathbf{t})\right)-\mathbf{G}\left(\boldsymbol{\mu}^{(\mathbf{1})}\left(\mathbf{t}-\frac{\Delta}{\mathbf{m}}\right), \xi^{(\mathbf{1})}\left(\mathbf{t}-\frac{\Delta}{\mathbf{m}}\right)\right)$,

where $m \geqslant 1$ is a sufficiently large integer. The delay is divided up into small fractions with duration $\frac{\Delta}{m}$ in such a way that asymptotic convergence to zero of the prediction error is guaranteed. Noticing that, by integrating over $\left[t-\frac{\Delta}{m}, t\right]$ the system equations (4) retarded by $\Delta-\frac{\Delta}{m}$ and since $x^{(1)}\left(t-\frac{\Delta}{m}\right)=x^{(0)}(t)$ by definition,

$x^{(1)}(t)=x^{(0)}(t)+\int_{t-\frac{\Delta}{m}}^{t}\left[A x^{(1)}(s)+\phi\left(x^{(1)}(s)\right)\right] d s$,

the predictor (11) is obtained by time-differentiating

$$
\xi^{(1)}(t)=\xi^{(0)}(t)+\int_{t-\frac{\Delta}{m}}^{t} M\left(\mu^{(1)}(s), \xi^{(1)}(s)\right) d s
$$

which is a copy of (13) with $A x^{(1)}+\phi\left(x^{(1)}\right)$ substituted for its saturated version $M\left(\mu^{(1)}, x^{(1)}\right)$. On the other hand, noticing that, by integrating over $\left[t-\frac{\Delta}{m}, t\right]$ the equations (9) retarded by $\Delta-\frac{\Delta}{m}$ and since $v^{(1)}(t-$ $\left.\frac{\Delta}{m}\right)=v^{(0)}(t)$ by definition,

$$
\begin{aligned}
& v^{(1)}(t)=v^{(0)}(t)+\int_{t-\frac{\Delta}{m}}^{t}\left[G\left(v^{(1)}(s), \xi^{(0)}\left(s+\frac{\Delta}{m}\right)\right)\right. \\
& \left.+Q\left(v^{(1)}(s), \xi^{(0)}\left(s+\frac{\Delta}{m}\right), y^{(1)}(s)\right)\right] d s
\end{aligned}
$$

(here, $y^{(1)}(\cdot)$ means $\left.y\left(\cdot+\frac{\Delta}{m}\right)\right)$, the predictor $(12)$ is obtained by time-differentiating a copy of (15) after ignoring the innovation term $Q$. The saturation of the estimates $\xi^{(1)}$ and the one-step prediction $\mu^{(1)}$ of the saturation level is a crucial issue in our design (see section 6).

In general, for each $j=2, \ldots, m$ the estimates $\xi^{(i)}(t)$ and $\mu^{(i)}(t), i=0, \ldots, j-1$, are used in the blocks devoted, on one hand, to the estimation $\xi^{(j)}(t)$ of $x^{(j)}(t), t \geqslant 0$, ( $j$-steps prediction)

$\dot{\xi}^{(\mathbf{j})}(\mathbf{t})=\dot{\xi}^{(\mathbf{j}-\mathbf{1})}(\mathbf{t})+\mathbf{M}\left(\boldsymbol{\mu}^{(\mathbf{j})}(\mathbf{t}), \xi^{(\mathbf{j})}(\mathbf{t})\right)$

$-\mathbf{M}\left(\mu^{(\mathbf{j})}\left(\mathbf{t}-\frac{\Delta}{\mathbf{m}}\right), \xi^{(\mathbf{j})}\left(\mathbf{t}-\frac{\Delta}{\mathbf{m}}\right)\right), \mathbf{j}=\mathbf{2}, \ldots, \mathbf{m}$,

and, on the other, to the estimation $\mu^{(j)}(t)$ of $v^{(j)}(t):=$ $\mu^{(0)}\left(t+j \frac{\Delta}{m}\right)$

$\dot{\boldsymbol{\mu}}^{(\mathbf{j})}(\mathbf{t})=\dot{\boldsymbol{\mu}}^{(\mathbf{j}-\mathbf{1})}(\mathbf{t})+\mathbf{G}\left(\boldsymbol{\mu}^{(\mathbf{j})}(\mathbf{t}), \boldsymbol{\xi}^{(\mathbf{j})}(\mathbf{t})\right)$

$-\mathbf{G}\left(\boldsymbol{\mu}^{(\mathbf{j})}\left(\mathbf{t}-\frac{\Delta}{\mathbf{m}}\right), \boldsymbol{\xi}^{(\mathbf{j})}\left(\mathbf{t}-\frac{\Delta}{\mathbf{m}}\right)\right), \mathbf{j}=\mathbf{2}, \ldots, \mathbf{m}$.
The saturation of the estimates $\xi^{(j)}$ and the $j$-steps prediction $\mu^{(j)}$ of the saturation level is a crucial issue in our design (see section 6). Overall, the predictor (7)-(9)(11)-(12)-(16)-(17) consists of $2(m+1)$ filters, each pair chained to the other, and it is initialized as follows: for all $j=1, \ldots, m$ and $s \in\left[-\frac{\Delta}{m}, 0\right]$

$$
\begin{aligned}
& \mu^{(0)}(0):=\mu_{0}^{(0)}:=\hat{\mu}(-\Delta), \\
& \mu^{(j)}(s):=\mu_{0}^{(j)}(s):=\hat{\mu}\left(s-\Delta+\frac{j \Delta}{m}\right), \\
& \xi^{(0)}(0):=\xi_{0}^{(0)}:=\hat{\xi}(-\Delta), \\
& \xi^{(j)}(s):=\xi_{0}^{(j)}(s):=\hat{\xi}\left(s-\Delta+\frac{j \Delta}{m}\right),
\end{aligned}
$$

with bounded $\hat{\xi} \in C^{0}\left([-\Delta, 0], \mathbb{R}^{n}\right)$ and $\hat{\mu} \in C^{0}([-\Delta, 0]$, $[1,+\infty))$. For simplicity, we can assume $\hat{\xi} \equiv 0$ and $\hat{\mu} \equiv$ 1 .The vector of the initial conditions $\left(x_{0}, \hat{\mu}, \hat{\xi}\right)$ will be denoted in what follows by $\varphi_{0}$.

We want to prove that the estimates $\xi^{(j)}(t)$ converge to the actual delayed states $x^{(j)}(t)$ for $j=0, \ldots, m$, and most importantly, convergence of $\xi^{(m)}(t)$ to the undelayed state $x(t)$. The main result of this paper is the following.

Theorem 3 Assume HO, H1 and H2. There exist c, $K>$ 0 , integer $m \geqslant 1$ and diagonal positive definite $\Gamma \in \mathbb{R}^{n \times n}$ such that the solution $x\left(\cdot, x_{0}\right), \xi^{(j)}\left(\cdot, \varphi_{0}\right), \mu^{(j)}\left(\cdot, \varphi_{0}\right), j=$ $0, \ldots, m$, of (4) with (7)-(9)-(11)-(12)-(16)-(17) is defined and bounded for all times and initial conditions $\varphi_{0}$. Moreover, $\lim _{t \rightarrow \infty}\left\|x\left(t, x_{0}\right)-\xi^{(m)}\left(t, \varphi_{0}\right)\right\|=0$.

Remark 4 Theorem 3 can be directly extended to systems (4) with $m$ inputs and $p$ measurements and $\phi$ substituted for $\phi+B F+D$, where $(A, B)$ are block diagonal with $p$ diagonal blocks in Brunowski form, $C$ with $p$ diagonal blocks $C_{i}=(1,0, \ldots, 0), F \in \mathbb{R}^{p \times n}$ and diagonal $D \in \mathbb{R}^{n \times n}$.

Remark 5 The predictor (7)-(9)-(11)-(12)-(16)-(17) is robust with respect to square integrable output disturbances. Also robustness with respect to non-vanishing output disturbances can be achieved by suitably modifying the predictor (this will be the object of future work).

Before proving our main result (theorem 3) we outline the constructive steps for the predictor (7)-(9)-(11)-(12)(16)-(17).

\section{Constructive design of the predictor}

The predictor (7)-(9)-(11)-(12)-(16)-(17) is characterized, besides its initial conditions which have been specified in (18), by $c, K, m$ and $\Gamma$. These quantities are chosen as follows. Let $\Phi, \Psi$ and $\mathfrak{g}$ be as in assumption $H 0$. 
(a) $K>0$ and the diagonal positive definite $\Gamma \in \mathbb{R}^{n \times n}$ are such that ${ }^{1}$

$0<\mathfrak{S}:=2\left(K C^{T} C+A^{T} \Gamma A\right)$

$-\left(A+A^{T} \Gamma^{2}\right)\left(I-A^{T} \Gamma\right)^{-1}-\left(I-A^{T} \Gamma\right)^{-T}\left(A+A^{T} \Gamma^{2}\right)^{T}$

(b) $c, \alpha>0$ are such that if $\Phi^{M}, \Psi^{M} \in \mathbb{R}^{n \times n}$ are matrices for which

$\Phi\left(w^{\prime}, z^{\prime}\right) \leq \Phi^{M}, \forall w^{\prime}, z^{\prime} \in \mathbb{R}^{n}:\left\|z^{\prime}\right\| \leqslant n c,\left\|w^{\prime}\right\| \leqslant n c(20)$

$\Psi\left(w^{\prime}, z^{\prime}\right) \leq \Psi^{M}, \forall w^{\prime}, z^{\prime} \in \mathbb{R}^{n}:\left\|z^{\prime}\right\| \leqslant n c,\left\|w^{\prime}\right\| \leqslant n c$

(we recall that $\leq$ for matrices means $\leqslant$ for each entry) then $^{2}$

$$
\begin{aligned}
& \alpha I<\mathfrak{S}-\left(2\left(I+A^{T} \Gamma\right) \Phi^{M}+2 K C^{T} \Psi^{M}\right)\left(I-A^{T} \Gamma\right)^{-1} \\
& -\left(I-A^{T} \Gamma\right)^{-T}\left(2\left(I+A^{T} \Gamma\right) \Phi^{M}+2 K C^{T} \Psi^{M}\right)^{T} \\
& -4 n^{2} c^{2} \max _{i}\left|\mathfrak{g}_{i}\right|\left[A^{T} \Gamma\left(I-A^{T} \Gamma\right)^{-1}+\left(I-A^{T} \Gamma\right)^{-T} \Gamma A\right]
\end{aligned}
$$

(c) $m$ is an integer such that

$\Delta\left[\|A\|+n^{2} c^{2}\right]<m$.

\section{Examples and simulations}

The system

$$
\begin{aligned}
& \dot{x}_{1}=x_{2} \\
& \dot{x}_{2}=\phi_{2}\left(x_{1}, x_{2}\right):=-x_{1}+\left(1-x_{1}^{2} x_{2}^{2}\right) x_{2}
\end{aligned}
$$

with delayed measurement $y=x_{1}(t-\Delta)$, satisfies assumptions $H 0, H 1, H 2$ of theorem 6 . A predictor has been designed according to our procedure and a simulation has been worked out with delay $\Delta=1$ and initial conditions $x(-\Delta)=(10,5)^{T}, \hat{\xi}(s)=(0,0)^{T}$ and

1 Inequality (19) is solved on account of the fact that $\mathfrak{S}$ can be defined recursively as $\mathfrak{S}:=\mathfrak{S}^{(n)}$ where (recall that $\Gamma_{i j}$ denotes the $(i, j)$ entry of $\Gamma)$

$$
\begin{gathered}
\mathfrak{S}^{(j)}:=\left[\begin{array}{c|c}
2 \Gamma_{n-j, n-j}+\mathfrak{N}^{(j-1)} & \left(\mathfrak{M}^{(j-1)}\right)^{T} \\
\hline \mathfrak{M}^{(j-1)} & \mathfrak{S}^{(j-1)}
\end{array}\right], \\
j=2, \ldots, n, \mathfrak{S}^{(1)}:=2 \Gamma_{n-1, n-1}, \quad \Gamma_{00}:=K,
\end{gathered}
$$

and $\mathfrak{N}^{(j-1)} \quad \in \quad \mathbf{C}^{0}\left(\mathbb{R}^{j-1}, \mathbb{R}\right) \quad$ and $\quad \mathfrak{M}^{(j-1)} \quad \in$ $\mathbf{C}^{0}\left(\mathbb{R}^{j-1}, \mathbb{R}^{j-1}\right), j=2, \ldots, n$, are suitable functions of $\Gamma_{n-j+1, n-j+1}, \ldots, \Gamma_{n-1, n-1}$. Therefore, it is sufficient to pick any $\Gamma_{n-1, n-1}>0$ and for each increasing $j=2, \ldots, n$ select $\Gamma_{n-j, n-j}>0$ such that $\mathfrak{S}^{(j)}>0$.

2 The numbers $c, \alpha$ always exist on account of (19) and since $\Phi$ and $\Psi$ are continuous and $\Phi(0,0)=0$ and $\Psi(0,0)=0$ (assumption $\mathrm{HO}$ ). $\hat{\mu}(s)=1$ for all $s \in[-\Delta, 0]$. The prediction of $x(t)$ is worked out through the intermediate predictions of the delayed states $x(t-.66)$ and $x(t-.33)$ (we chose $m=3$, i.e. the delay period is divided into three subintervals). In particular, $x(t-1)$ is estimated through the observer (7)-(9) while $x(t-.66), x(t-.33)$ and $x(t)$ are estimated through the chained predictors (16)-(17). The saturation levels of the estimates are set to $c \mu^{\mathfrak{r}}$ with $c=.5$ and $\mathfrak{r}_{1}=1$ and $\mathfrak{r}_{2}=3$. The prediction errors for $x(t)$ are shown in Fig. 1.

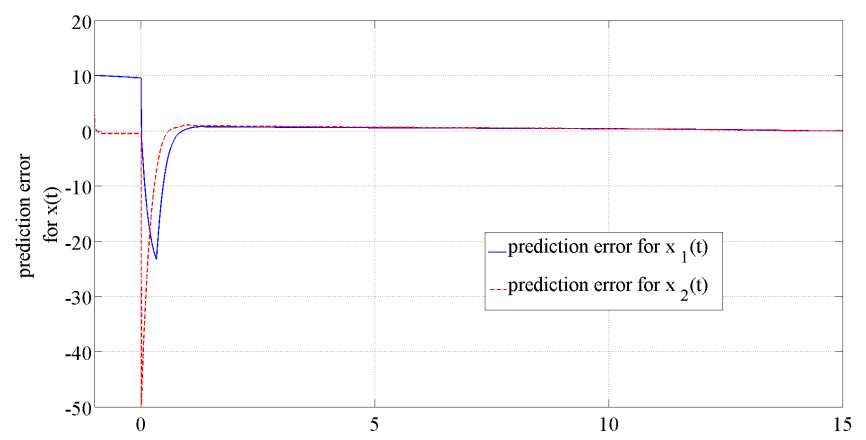

Fig. 1. Prediction errors for $x(t)$ with saturated estimates and saturation level prediction $\left(x(-\Delta)=(10,5)^{T}\right)$.

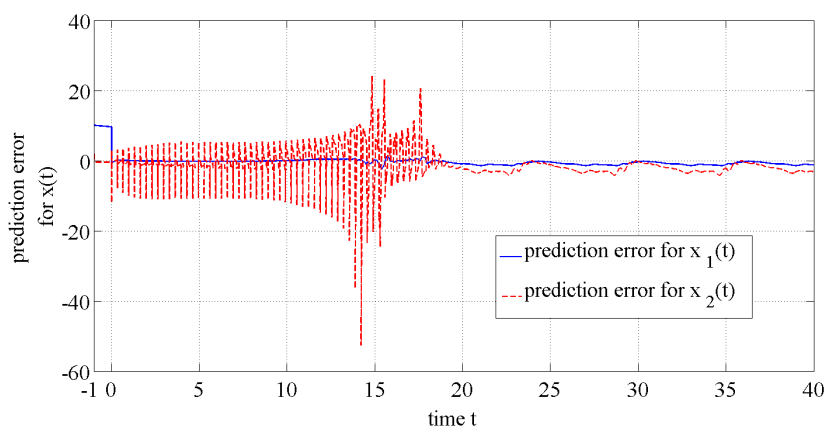

Fig. 2. Prediction errors for $x(t)$ using predictors without saturated estimates and saturation level prediction $\left(x(-\Delta)=(10,5)^{T}\right)$.

The saturations of the predictions and the predictions of the saturation levels at time $t-.66, t-.33$ and $t$ in the chained predictors (16)-(17) are a crucial issue in the predictor design. Also, the numbers $\mathfrak{r}_{1}$ and $\mathfrak{r}_{2}$ according to which the saturation levels are differently weighted $\left(\mu^{\mathfrak{r}_{1}}\right.$ and, resp., $\left.\mu^{\mathfrak{r}_{2}}\right)$ and which represent the weights associated with the incremental homogeneity degrees $\mathfrak{g}_{1}$ and $\mathfrak{g}_{2}$ of the nonlinearity of (24) are crucial for a correct prediction of $x(t)$. Indeed, a simulation has been worked out by implementing the prediction of $x(t-.66)$, $x(t-.33)$ and $x(t)$ without saturations and saturation levels prediction, i.e. simple prediction steps. Due to the large initial state values $x(-\Delta)$ and the strong nonlinearity $x_{1}^{2} x_{2}^{3}$ in (24), the prediction error dynamics has a non-zero attractor (Fig. 2). Notice that there is a nonzero steady-state prediction error despite the fact that 
the steady-state estimation error for the undelayed state $x(t-1)$ is zero (Fig.3). This shows that a nonlinear observer for the undelayed state $x(t-1)$ (such as $(7)-(9))$ cascaded with simple prediction steps (i.e. without saturations and saturation levels prediction) is not sufficient for correctly predicting the state at delayed time instants. A theoretical explanation for this fact is that using simple prediction steps, i.e. without saturations and level saturation prediction, such as

$$
\begin{aligned}
& \dot{\xi}^{(j)}(t)=\dot{\xi}^{(j-1)}(t)+A \xi^{(j)}(t)+\phi\left(\xi^{(j)}(t)\right) \\
& -\left[A \xi^{(j)}\left(t-\frac{\Delta}{m}\right)+\phi\left(\xi^{(j)}\left(t-\frac{\Delta}{m}\right)\right)\right], j=2, \ldots, m,
\end{aligned}
$$

the $j$-steps prediction error $e^{(j)}(t)$ satisfies an integral inequality $\left\|e^{(j)}(t)\right\| \leqslant \beta^{(j)}(t)+\alpha^{(j)} \int_{t-\frac{\Delta}{m}}^{t} \gamma^{(j)}\left(\left\|e^{(j)}(\theta)\right\|\right) d \theta$ where $\gamma^{(j)}(\cdot)$ is an increasing locally Lipschitz function such that $\gamma^{(j)}(0)=0$, and $\alpha^{(j)}>0$ and $\beta^{(j)}(\cdot)$ depend on the initial conditions $x(-\Delta)$ with $\lim _{t \rightarrow+\infty} \beta^{(j)}(t)=0$. In the case of our example, $\gamma^{(j)}(s)=s\left(1+s^{4}\right)$. The prediction error $e^{(j)}(t)$ is not guaranteed to tend to zero as $t \rightarrow+\infty$ unless $m$ is such that $\frac{\Delta}{m} \gamma^{(j)}\left(\sup _{\theta \in\left[t-\frac{\Delta}{m}, t\right]}\left\|e^{(j)}(\theta)\right\|\right) \leqslant\left\|e^{(j)}(t)\right\|$ for all $t \geqslant 0$. This requires $m$ to be large when the prediction error is large, which is not feasible with an integer $m$ since the prediction error depends on the unknown $x(-\Delta)$ and, moreover, for large values of the prediction error the number of the chained predictors (16)-(17) would be very high. By introducing saturations and level saturation prediction as pointed out in (16)-(17) (and using the incremental homogeneity properties of the nonlinearities) the function $\gamma^{(j)}$ is linear and $e^{(j)}(t)$ tends to zero as $t \rightarrow+\infty$.

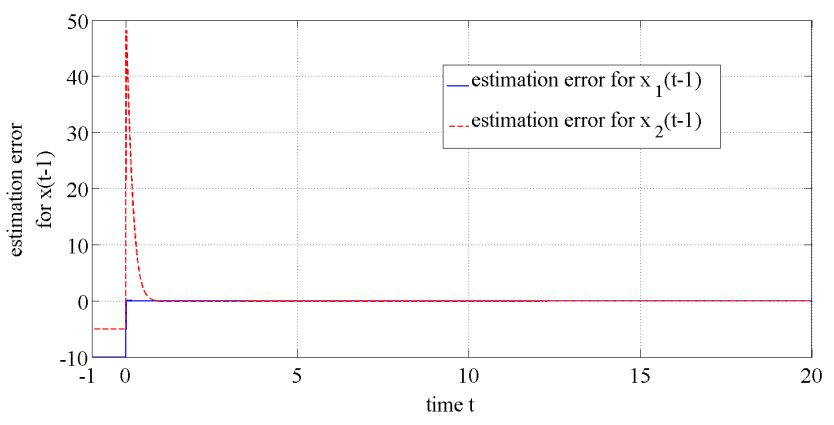

Fig. 3. Estimation errors for the undelayed state $x(t-1)$ with unsaturated predictions $\left(x(-\Delta)=(10,5)^{T}\right)$.

\section{$7 \quad$ Proof of the main result}

In order to prove theorem 3 we first prove that $\xi^{(0)}(t)$ converges to the delayed state $x^{(0)}(t):=x(t-\Delta)$ and, secondly, that $\xi^{(j)}(t)$ converges to the delayed state $x^{(j)}(t):=x\left(t-\Delta+j \frac{\Delta}{m}\right)$ for $j=1, \ldots, m$.

\subsection{The observer for $x^{(0)}$}

The state $x^{(0)}(\cdot):=x(\cdot-\Delta)$ satisfies the equations

$$
\dot{x}^{(0)}=A x^{(0)}+\phi\left(x^{(0)}\right), y=C x^{(0)}+\psi\left(x^{(0)}\right),
$$

which we consider together with the first block equations (7)-(9) of the predictor. The vector of the initial conditions $x_{0}^{(0)}, \mu_{0}^{(0)}$ and $\xi_{0}^{(0)}$ will be denoted in what follows by $\varphi_{0}^{(0)}$. Wherever necessary we will maintain the explicit dependence of the state trajectories from the initial conditions.

Proposition 6 Assume H0, H1 and H2. Let c, $\alpha, K>0$ and $\Gamma, \Phi^{M}, \Psi^{M} \in \mathbb{R}^{n \times n}$ be as in section 5 . The solution $x^{(0)}\left(\cdot, x_{0}\right), \xi^{(0)}\left(\cdot, \varphi_{0}^{(0)}\right)$ and $\mu^{(0)}\left(\cdot, \varphi_{0}^{(0)}\right)$ of $(26)-(7)-(9)$ is defined and bounded for all times and initial conditions $\varphi_{0}^{(0)}$. Moreover, $\lim _{t \rightarrow \infty}\left\|x^{(0)}\left(t, x_{0}\right)-\xi^{(0)}\left(t, \varphi_{0}^{(0)}\right)\right\|=0$.

PROOF. Throughout the proof, we will omit the superscript $(0)$. Let $e:=x-\xi$ be the estimation error. Therefore, the state equations (26)-(7)-(9) read out as

$$
\begin{aligned}
\dot{x} & =A x+\phi(x), \\
\dot{e} & =[A-L(\mu) C] e+\gamma(\mu, x)-\gamma\left(\mu, \sigma\left(c \mu^{\mathfrak{r}},-e+x\right)\right), \\
\dot{\mu} & =G(\mu,-e+x)+Q(\mu,-e+x, y),
\end{aligned}
$$

where $\gamma(\mu, x):=\phi(x)-L(\mu) \psi(x)$. First of all, notice that for how we set the initial condition for $\mu(t)$ in (18) (i.e. $\mu(0):=\hat{\mu}(-\Delta)$ with $\left.\hat{\mu} \in C^{0}([-\Delta, 0],[1,+\infty))\right)$ and since $G$ and $Q$ are non-negative functions of their arguments, $\mu(t) \geqslant 1$ for all $t \geqslant 0$. Perform the following coordinate transformation

$$
\begin{aligned}
& (x, e, \mu) \mapsto(x, \eta, \mu): \\
& \eta:=\mathfrak{X}^{-1}(\mu) e, \mathfrak{X}(\mu):=\left(I-A^{T} \mathfrak{G}(\mu)\right)^{-1}
\end{aligned}
$$

Recalling that

$\mathfrak{G}(\mu):=\operatorname{diag}\left\{\Gamma \mu^{2 A \mathfrak{g}}\right\}, L(\mu):=\mathfrak{X}(\mu) C^{T} K \mu^{2 C \mathfrak{g}}$

and using the identities

$C C^{T}=1, A^{T} \mathfrak{G}(\mu) A C^{T}=0, C \mathfrak{X}(\mu)=C$

$A^{T} \mathfrak{G}(\mu) A A^{T}=A^{T} \mathfrak{G}(\mu), \mathfrak{X}(\mu)-I=A^{T} \mathfrak{G}(\mu) \mathfrak{X}(\mu)$

and $\frac{d}{d \mu}\left(A^{T} \mathfrak{G}(\mu)\right)=\frac{2}{\mu} \operatorname{diag}\left\{A^{T} A \mathfrak{g}\right\} A^{T} \mathfrak{G}(\mu),(27)$ reads out in the new coordinates

$$
\begin{aligned}
\dot{x} & =A x+\phi(x), \\
\dot{\eta} & =-\mathfrak{H}(\mu) \eta-2 \frac{\dot{\mu}}{\mu} \operatorname{diag}\left\{A^{T} A \mathfrak{g}\right\} A^{T} \mathfrak{G}(\mu) \mathfrak{X}(\mu) \eta, \\
& +\mathfrak{Z}\left(\mu, \mathfrak{X}^{-1}(\mu) x\right)-\mathfrak{Z}\left(\mu,-\eta+\mathfrak{X}^{-1}(\mu) x\right)+\rho(\mu, x), \\
\dot{\mu} & =G(\mu,-\mathfrak{X}(\mu) \eta+x)+Q(\mu,-\mathfrak{X}(\mu) \eta+x, y),
\end{aligned}
$$


with

$$
\begin{aligned}
& \mathfrak{H}(\mu):=C^{T} K \mu^{2 C \mathfrak{g}} C+A^{T} \mathfrak{G}(\mu) A \\
& \mathfrak{Z}(\mu, z):=\left(I-\mathfrak{H}(\mu) A^{T}\right) \phi\left(\sigma\left(c \mu^{\mathfrak{r}}, \mathfrak{X}(\mu) z\right)\right) \\
& -\mathfrak{H}(\mu) C^{T} \psi\left(\sigma\left(c \mu^{\mathfrak{r}}, \mathfrak{X}(\mu) z\right)\right)+\left[A-A^{T} \mathfrak{G}^{2}(\mu)\right] \mathfrak{X}(\mu) z \\
& \rho(\mu, x):=\left(I-\mathfrak{H}(\mu) A^{T}\right)\left[\phi(x)-\phi\left(\sigma\left(c \mu^{\mathfrak{r}}, x\right)\right)\right] \\
& -\mathfrak{H}(\mu) C^{T}\left[\psi(x)-\psi\left(\sigma\left(c \mu^{\mathfrak{r}}, x\right)\right)\right]
\end{aligned}
$$

By (i) and (ii) of lemma 11 and the definition of incremental homogeneity in the upper bound, for all $\mu \geqslant 1$ and $w, z \in \mathbb{R}^{n}$

$\mathfrak{H}(\mu)\left(\mu^{\mathfrak{r}} \diamond z\right)=\mu^{\mathfrak{r}+\mathfrak{g}} \diamond\left(H\left(\mu^{\mathfrak{g}} \diamond z\right)\right)$

$\left\langle\left\langle\mathfrak{Z}\left(\mu, \mu^{\mathfrak{r}} \diamond w\right)-\mathfrak{Z}\left(\mu, \mu^{\mathfrak{r}} \diamond z\right)\right\rangle\right\rangle \leq \mu^{\mathfrak{r}+\mathfrak{g}} \diamond\left(\Omega\left\langle\left\langle\mu^{\mathfrak{g}} \diamond(w-z)\right\rangle\right)\right.$

(recall that $\leq$ means $\leqslant$ componentwise and $\langle\langle\cdot\rangle\rangle$ means $|\cdot|$ component wise: see notation section) where

$H:=C^{T} K C+A^{T} \Gamma A$

$\Omega:=\left[2\left(I+H A^{T}\right) \Phi^{M}+2 H C^{T} \Psi^{M}+A+A^{T} \Gamma^{2}\right] X(36)$

$X:=\left(I-A^{T} \Gamma\right)^{-1}$,

and $\Phi^{M}, \Psi^{M} \in \mathbb{R}^{n \times n}$ are the matrices selected in (20)(21).

Let $V(\mu, \eta):=\left\|\mu^{-\mathfrak{r}} \diamond \eta\right\|^{2}$. We evaluate the derivative of $V$ along the trajectories of (30). To this aim, notice on account of (33) (with $z:=\mu^{-\mathfrak{r}} \diamond \eta$ ) and, resp., of (34) $\left(\right.$ with $w:=\mu^{-\mathfrak{r}} \diamond \mathfrak{X}^{-1}(\mu) x$ and $z:=\mu^{-\mathfrak{r}} \diamond\left(-\eta+\mathfrak{X}^{-1}(\mu) x\right)$ ) and using the properties (2)-(3)

$$
\begin{aligned}
& \frac{\partial V}{\partial \eta}(\mu, \eta)\left\{-\mathfrak{H}(\mu) \eta+\mathfrak{Z}\left(\mu, \mathfrak{X}^{-1}(\mu) x\right)-\mathfrak{Z}\left(\mu,-\eta+\mathfrak{X}^{-1}(\mu) x\right)\right\} \\
& =-2\left(\mu^{-\mathfrak{r}} \diamond \mu^{-\mathfrak{r}} \diamond \eta\right)^{T} \mathfrak{H}(\mu)\left(\mu^{\mathfrak{r}} \diamond \mu^{-\mathfrak{r}} \diamond \eta\right) \\
& +2\left(\mu^{-\mathfrak{r}} \diamond \mu^{-\mathfrak{r}} \diamond \eta\right)^{T}\left\{\mathfrak{Z}\left(\mu, \mu^{\mathfrak{r}} \diamond \mu^{-\mathfrak{r}} \diamond \mathfrak{X}^{-1}(\mu) x\right)\right. \\
& \left.-\mathfrak{Z}\left(\mu, \mu^{\mathfrak{r}} \diamond \mu^{-\mathfrak{r}} \diamond\left(-\eta+\mathfrak{X}^{-1}(\mu) x\right)\right)\right\} \\
& \leqslant-2\left(\mu^{-\mathfrak{r}} \diamond \eta\right)^{T}\left(\mu^{-\mathfrak{r}} \diamond \mathfrak{H}(\mu)\left(\mu^{\mathfrak{r}} \diamond \mu^{-\mathfrak{r}} \diamond \eta\right)\right) \\
& +2\left\langle\left\langle\mu^{-\mathfrak{r}} \diamond \eta\right\rangle\right\rangle^{T}\left(\mu ^ { - \mathfrak { r } } \diamond \left\langle\left\langle\mathfrak{Z}\left(\mu, \mu^{\mathfrak{r}} \diamond \mu^{-\mathfrak{r}} \diamond \mathfrak{X}^{-1}(\mu) x\right)\right.\right.\right. \\
& \left.\left.-\mathfrak{Z}\left(\mu, \mu^{\mathfrak{r}} \diamond \mu^{-\mathfrak{r}} \diamond\left(-\eta+\mathfrak{X}^{-1}(\mu) x\right)\right)\right\rangle\right) \\
& \leqslant-\left\langle\left\langle\mu^{-\mathfrak{r}+\mathfrak{g}} \diamond \eta\right\rangle\right)^{T}\left[2 H-\Omega-\Omega^{T}\right]\left\langle\left\langle\mu^{-\mathfrak{r}+\mathfrak{g}} \diamond \eta\right\rangle\right\rangle
\end{aligned}
$$

Moreover, since $\left\|\mu^{-\mathfrak{r}} \diamond \sigma\left(c \mu^{\mathfrak{r}}, w\right)\right\| \leqslant n c$ for all $\mu \geqslant 1$ and $w \in \mathbb{R}^{n}$, recalling the definition of $Q$ and $G$ in (10) and that $\mu(t) \geqslant 1$ for all $t \geqslant 0$,

$$
\left|\frac{\dot{\mu}}{\mu}\right| \leqslant \frac{2 n^{2} c^{2}}{\mu^{2\left|\min _{i} \mathfrak{g}_{i}\right|}} \leqslant 2 n^{2} c^{2} \mu^{2 \min _{i} \mathfrak{g}_{i}}
$$

Since $\left\langle\left\langle\mu^{-\mathfrak{r}-\mathfrak{g}} \diamond \eta\right\rangle\right\rangle \leq \mu^{-2 \min _{i} \mathfrak{g}_{i}}\left\langle\left\langle\mu^{-\mathfrak{r}+\mathfrak{g}} \diamond \eta\right\rangle\right.$ for all $\mu \geqslant 1$ and $\eta \in \mathbb{R}^{n}$ and $(\mu, \eta) \mapsto A^{T} \mathfrak{G}(\mu) \mathfrak{X}(\mu) \eta$ is i.h.u.b. with quadruple $\left(\mathfrak{r}, \mathfrak{r}-\mathfrak{g}, \mathfrak{g}, A^{T} \Gamma X\right)((\mathrm{v})$ of lemma 11), using the properties (2)-(3)

$$
\begin{aligned}
& \left|\frac{\partial V}{\partial \eta}(\mu, \eta) \operatorname{diag}\left\{A^{T} A \mathfrak{g}\right\} A^{T} \mathfrak{G}(\mu) \mathfrak{X}(\mu) \eta\right|= \\
& \left|2\left(\mu^{-\mathfrak{r}} \diamond \mu^{-\mathfrak{r}} \diamond \eta\right)^{T} \operatorname{diag}\left\{A^{T} A \mathfrak{g}\right\} A^{T} \mathfrak{G}(\mu) \mathfrak{X}(\mu)\left(\mu^{\mathfrak{r}} \diamond \mu^{-\mathfrak{r}} \diamond \eta\right)\right| \\
& \leqslant 2 \max _{i}\left|\mathfrak{g}_{i}\right|\left\langle\left\langle\mu^{-\mathfrak{r}-\mathfrak{g}} \diamond \eta\right\rangle\right\rangle^{T} A^{T} \Gamma X\left\langle\left\langle\mu^{-\mathfrak{r}+\mathfrak{g}} \diamond \eta\right\rangle\right\rangle \\
& \leqslant \frac{\max _{i}\left|\mathfrak{g}_{i}\right|}{\mu^{2 \min _{i} \mathfrak{g}_{i}}}\left\langle\left\langle\mu^{-\mathfrak{r}+\mathfrak{g}_{\diamond}}\right\rangle\right\rangle^{T}\left[A^{T} \Gamma X+X^{T} \Gamma A\right]\left\langle\left\langle\mu^{-\mathfrak{r}+\mathfrak{g}} \diamond \eta\right\rangle\right\rangle
\end{aligned}
$$

Moreover, by Young(/Peter-Paul)'s inequality and using the properties (2)-(3), for all $\alpha>0$

$\left|\frac{\partial V}{\partial \eta}(\mu, \eta) \rho(\mu, x)\right| \leqslant \frac{\alpha}{2}\left\|\mu^{-\mathfrak{r}+\mathfrak{g}} \diamond \eta\right\|^{2}+\frac{2}{\alpha}\left\|\mu^{-\mathfrak{r}-\mathfrak{g}} \diamond \rho(\mu, x)\right\|^{2}$

Collecting this with inequalities (38) and (40) and using (22) (upon noting that the left-hand part of the inequality $(22)$ is equal to $2 H-\Omega-\Omega^{T}-$ $\left.4 n^{2} c^{2} \max _{i}\left|\mathfrak{g}_{i}\right|\left[A^{T} \Gamma X+X^{T} \Gamma A\right]\right)$

$$
\left.\dot{V}\right|_{(30)} \leqslant-\frac{\alpha}{2}\left\|\mu^{-\mathfrak{r}+\mathfrak{g}} \diamond \eta\right\|^{2}+\frac{2}{\alpha}\left\|\mu^{-\mathfrak{r}-\mathfrak{g}} \diamond \rho(\mu, x)\right\|^{2}
$$

To conclude the proof, we prove that $\mu(t)$ is bounded (Claim \#1 below) and $y(t)-Y(\mu(t), \xi(t))$ and $\sigma\left(c \mu^{\mathfrak{r}}(t), \xi(t)\right)-\xi(t)$ tend asymptotically to zero (Claim \#2 below), which, as we will see, by assumption H2 implies that also $e(t)$ tend asymptotically to zero. In other words, the estimates $\xi$ tend to de-saturate and the innovation $L(\mu)(y-Y(\mu, \xi))$ in the observer $(7)$ tend to zero.

Claim \#1. $1 \leqslant \mu(t)<+\infty$ for all $t \geqslant 0$

We already established that $1 \leqslant \mu(t)$ for all $t \geqslant 0$. Since $\dot{\mu}(t) \geqslant 0$ for all $t \geqslant 0$, there clearly exists $T_{\varphi_{0}} \geqslant$ 0 (depending on the initial conditions $\varphi_{0}$ ) such that $\lim _{t \rightarrow T_{\varphi_{0}}^{-}} \mu(t)=\mu_{\infty} \leqslant+\infty$ with $\left[0, T_{\varphi_{0}}\right)$ being the right maximum extension interval of $\mu(t)$. Assume by absurd that $\mu_{\infty}=+\infty$. By $H 1$ there exists $T_{\varphi_{0}}^{\prime} \in\left[0, T_{\varphi_{0}}\right)$ such that $\langle\langle x(t)\rangle\rangle \leq c \mu^{\mathfrak{r}}(t)$ for all $t \in\left[T_{\varphi_{0}}^{\prime}, T_{\varphi_{0}}\right)$ and, therefore, on the same time interval

$\sigma\left(c \mu^{\mathfrak{r}}(t), x(t)\right)=x(t)$

for which it follows that $\rho(\mu(t), x(t))=0$ for $t \in$ $\left[T_{\varphi_{0}}^{\prime}, T_{\varphi_{0}}\right)$. Since $\dot{\mu}(t)$ and $\mu(t)$ are non-negative for all $t \in\left[T_{\varphi_{0}}^{\prime}, T_{\varphi_{0}}\right)$, by integrating (41) over the interval $\left[T_{\varphi_{0}}^{\prime}, t\right]$ for each $t \in\left[T_{\varphi_{0}}^{\prime}, T_{\varphi_{0}}\right)$

$\int_{T_{\varphi_{0}}^{\prime}}^{t}\left\|\mu^{-\mathfrak{r}+\mathfrak{g}}(\theta) \diamond \eta(\theta)\right\|^{2} d \theta \leqslant \frac{2}{\alpha}\left\|\mu^{-\mathfrak{r}}\left(T_{\varphi_{0}}^{\prime}\right) \diamond \eta\left(T_{\varphi_{0}}^{\prime}\right)\right\|^{2}(43)$ 
By (ii) of lemma 9 and since $\mu^{-\left|\min _{i} \mathfrak{g}_{i}\right|} \mu^{-\mathfrak{r}} \leq \mu^{-\mathfrak{r}+\mathfrak{g}}$ for all $\mu \geqslant 1$, using (42) and recalling the definition of $Q$ and $G$ in $(10)$ and that $\mu(t) \geqslant 1$ for all $t \geqslant 0$,

$$
\begin{aligned}
& \frac{\dot{\mu}}{\mu}=\mu^{-2\left|\min _{i} \mathfrak{g}_{i}\right|}\left[\| \mu^{-\mathfrak{r}} \diamond \sigma\left(c \mu^{\mathfrak{r}},-\mathfrak{X}(\mu) \eta+x\right.\right. \\
& \left.-\sigma\left(c \mu^{\mathfrak{r}},-\mathfrak{X}(\mu) \eta+x\right)\right)\left\|^{2}+\right\| \mu^{-\mathfrak{r}} \diamond \sigma\left(c \mu^{\mathfrak{r}}, C^{T}(C \mathfrak{X}(\mu) \eta\right. \\
& \left.\left.+\psi(x)-\psi\left(\sigma\left(c \mu^{\mathfrak{r}},-\mathfrak{X}(\mu) \eta+x\right)\right)\right) \|^{2}\right] \\
& =\mu^{-2\left|\min _{i} \mathfrak{g}_{i}\right|}\left[\| \mu^{-\mathfrak{r}} \diamond \sigma\left(c \mu^{\mathfrak{r}},-\mathfrak{X}(\mu) \eta+\sigma\left(c \mu^{\mathfrak{r}}, x\right)\right.\right. \\
& \left.-\sigma\left(c \mu^{\mathfrak{r}},-\mathfrak{X}(\mu) \eta+x\right)\right)\left\|^{2}+\right\| \mu^{-\mathfrak{r}} \diamond \sigma\left(c \mu^{\mathfrak{r}}, C^{T}(C \mathfrak{X}(\mu) \eta\right. \\
& \left.\left.+\psi\left(\sigma\left(c \mu^{\mathfrak{r}}, x\right)\right)-\psi\left(\sigma\left(c \mu^{\mathfrak{r}},-\mathfrak{X}(\mu) \eta+x\right)\right)\right) \|^{2}\right] \\
& \leqslant \| \mu^{-\mathfrak{r}+\mathfrak{g}} \diamond\left\langle\left\langle\lambda_{1}\left(\mu, \mathfrak{X}^{-1}(\mu) x\right)-\lambda_{1}\left(\mu,-\eta+\mathfrak{X}^{-1}(\mu) x\right)\right\rangle \|^{2}\right. \\
& +\| \mu^{-\mathfrak{r}+\mathfrak{g}} \diamond\left\langle\left\langle\lambda_{2}\left(\mu, \mathfrak{X}^{-1}(\mu) x\right)-\lambda_{2}\left(\mu,-\eta+\mathfrak{X}^{-1}(\mu) x\right)\right\rangle \|^{2}\right.
\end{aligned}
$$

where

$$
\begin{aligned}
& \lambda_{1}(\mu, z):=-\mathfrak{X}(\mu) z+\sigma\left(c \mu^{\mathfrak{r}}, \mathfrak{X}(\mu) z\right), \\
& \lambda_{2}(\mu, z):=C^{T}\left(C \mathfrak{X}(\mu) z+\psi\left(\sigma\left(c \mu^{\mathfrak{r}}, \mathfrak{X}(\mu) z\right)\right)\right)
\end{aligned}
$$

But on account of (iii) of lemma 11 and the definition of incremental homogeneity in the upper bound, using the properties (2)-(3)

$$
\begin{aligned}
& \left\langle\left\langle\lambda_{1}\left(\mu, \mathfrak{X}^{-1}(\mu) x\right)-\lambda_{1}\left(\mu,-\eta+\mathfrak{X}^{-1}(\mu) x\right)\right\rangle\right\rangle \\
& \leq \mu^{\mathfrak{r}-\mathfrak{g}} \diamond\left\langle\left\langle 3 X\left(\mu^{-\mathfrak{r}+\mathfrak{g}} \diamond \eta\right)\right\rangle\right\rangle \\
& \left\langle\left\langle\lambda_{2}\left(\mu, \mathfrak{X}^{-1}(\mu) x\right)-\lambda_{2}\left(\mu,-\eta+\mathfrak{X}^{-1}(\mu) x\right)\right\rangle\right\rangle \\
& \leq \mu^{\mathfrak{r}-\mathfrak{g}} \diamond\left\langle\left\langle C^{T}\left(C+2 \Psi^{M}\right) X\left(\mu^{-\mathfrak{r}+\mathfrak{g}} \diamond \eta\right)\right\rangle\right\rangle
\end{aligned}
$$

By integration of $\frac{\dot{\mu}}{\mu}$ over $\left[T_{\varphi_{0}}^{\prime}, t\right]$ and collecting the above inequalities from (43) to (45), for all $t \in\left[T_{\varphi_{0}}^{\prime}, T_{\varphi_{0}}\right)$

$$
\begin{aligned}
& \ln \mu(t) \leqslant \ln \mu\left(T_{\varphi_{0}}^{\prime}\right)+\int_{T_{\varphi_{0}}^{\prime}}^{t}\left[\left\|3 X\left(\mu^{-\mathfrak{r}+\mathfrak{g}}(s) \diamond \eta(s)\right)\right\|^{2}\right. \\
& \left.+\left\|C^{T}\left(C+2 \Psi^{M}\right) X\left(\mu^{-\mathfrak{r}+\mathfrak{g}}(s) \diamond \eta(s)\right)\right\|^{2}\right] d s \\
& \leqslant \ln \mu\left(T_{\varphi_{0}}^{\prime}\right)+\frac{2}{\alpha}\left\|\mu^{-\mathfrak{r}}\left(T_{\varphi_{0}}^{\prime}\right) \diamond \eta\left(T_{\varphi_{0}}^{\prime}\right)\right\|^{2}\left[\|3 X\|^{2}\right. \\
& \left.+\left\|C^{T}\left(C+2 \Psi^{M}\right) X\right\|^{2}\right]<+\infty
\end{aligned}
$$

which gives a contradiction since $\lim _{t \rightarrow T_{\varphi_{0}}^{-}} \mu(t)=+\infty$. This proves our claim.

Claim \#2. $\lim _{t \rightarrow+\infty}[y(t)-Y(\mu(t), \xi(t))]=0$ and $\lim _{t \rightarrow+\infty}\left[\sigma\left(c \mu^{\mathfrak{r}}(t), \xi(t)\right)-\xi(t)\right]=0$.

By integrating $\frac{\dot{\mu}}{\mu^{1-2\left|\min _{i} \mathfrak{g}_{i}\right|}}$ over $[0,+\infty]$ and using claim \#1, we conclude that

$$
\begin{aligned}
& \kappa_{1}:=\mu^{-\mathfrak{r}} \diamond \sigma\left(c \mu^{\mathfrak{r}}, C^{T}[y-Y(\mu, \xi)]\right), \\
& \kappa_{2}:=\mu^{-\mathfrak{r}} \diamond \sigma\left(c \mu^{\mathfrak{r}}, \xi-\sigma\left(c \mu^{\mathfrak{r}}, \xi\right)\right) \in L^{2}\left(\mathbb{R}_{\geqslant}, \mathbb{R}^{n}\right)
\end{aligned}
$$

On the other hand, from (41) and boundedness of $\mu(t)$ (claim \#1) and $x(t)$ (assumption H1), since $\| \mu^{-\mathfrak{r}+\mathfrak{g}} \diamond$ $\eta \|^{2} \geqslant V(\mu, \eta) \mu^{2 \min _{i} \mathfrak{g}_{i}}$ for all $\eta$ and $\mu \geqslant 1$,

$$
\begin{aligned}
& \left.\dot{V}\right|_{(30)} \leqslant-V \frac{\alpha}{2} \min _{1 \leqslant \mu \leqslant \sup _{\theta \geqslant 0} \mu(\theta)} \mu^{2 \min _{i} \mathfrak{g}_{i}} \\
& +\frac{2}{\alpha} \max _{\substack{\|x\| \leqslant \sup _{\theta \geqslant 0}\|x(\theta)\| \\
1 \leqslant \mu \leqslant \sup _{\theta \geqslant 0} \mu(\theta)}}\left\|\mu^{-\mathfrak{r}-\mathfrak{g}} \diamond \rho(\mu, x)\right\|^{2}:=-a_{1} V+a_{2}
\end{aligned}
$$

for $a_{1}, a_{2}>0$, which implies that $V(\mu, \eta) \in L^{\infty}\left(\mathbb{R}_{\geqslant}, \mathbb{R}_{\geqslant}\right)$. Since $\|\eta\|^{2} \mu^{-2 \max _{i} \mathfrak{r}_{i}} \leqslant V(\mu, \eta)$ for all $\eta$ and $\mu \geqslant 1$ and by claim $\# 1$, we get $\eta \in L^{\infty}\left(\mathbb{R}_{\geqslant}, \mathbb{R}^{n}\right)$ and, therefore, since $e:=x-\xi:=\mathfrak{X}(\mu) \eta$, by claim \#1 and boundedness of $x(t)$ (assumption H1), also $e, \xi \in L^{\infty}\left(\mathbb{R}_{\geqslant}, \mathbb{R}^{n}\right)$ and

$$
\kappa_{1}, \kappa_{2} \in L^{\infty}\left(\mathbb{R}_{\geqslant}, \mathbb{R}^{n}\right)
$$

Moreover, since $x, \xi \in L^{\infty}\left(\mathbb{R}_{\geqslant}, \mathbb{R}^{n}\right)$ and by claim $\# 1$, also $\dot{x}, \dot{\xi} \in L^{\infty}\left(\mathbb{R}_{\geqslant}, \mathbb{R}^{n}\right), \dot{\mu} \in L^{\infty}\left(\mathbb{R}_{\geqslant}, \mathbb{R}_{\geqslant}\right)$and $x, \xi$ and $\mu$ are uniformly continuous over $\mathbb{R}_{\geqslant}$. If we prove that $\kappa_{1}, \kappa_{2}$ are uniformly continuous over $\mathbb{R}_{\geqslant}$, as a consequence of (46) and (48) we get $\lim _{t \rightarrow+\infty} \mathrm{K}_{1}(t)=0$ and $\lim _{t \rightarrow+\infty} \mathrm{K}_{2}(t)=$ 0 by virtue of Barbalat's lemma. Since $\mu^{-\mathfrak{r}} \diamond \sigma\left(c \mu^{\mathfrak{r}}, z\right)=$ 0 for each $\mu \geqslant 1$ if and only if $z=0$ and by virtue of claim \#1, we obtain claim \#2. Let's see that $\kappa_{2}$ is uniformly continuous over $\mathbb{R}_{\geqslant}$(for $\kappa_{1}$ similar arguments are used). Clearly $\mu^{-\mathfrak{r}}$ and $\mu^{\mathfrak{r}}$ are uniformly continuous over $\mathbb{R}_{\geqslant}$, being continuous with bounded derivative over $\mathbb{R}_{\geqslant}$, and $\sigma\left(c \mu^{\mathfrak{r}}, z\right)$ is uniformly continuous over $\mathbb{R}_{\geqslant}$for any uniformly continuous $z$ over $\mathbb{R}_{\geqslant}$: indeed, using (i) of lemma 9, lemma 10 and the triangle inequality, for each $k>0$ we always find $\delta, h_{1}, h_{2}>0$ such that for all $t_{2}, t_{1} \geqslant 0:\left|t_{2}-t_{1}\right| \leqslant \delta$

$\left\|\sigma\left(c \mu^{\mathfrak{r}}\left(t_{2}\right), z\left(t_{2}\right)\right)-\sigma\left(c \mu^{\mathfrak{r}}\left(t_{1}\right), z\left(t_{1}\right)\right)\right\|$

$\leqslant\left\|\sigma\left(c \mu^{\mathfrak{r}}\left(t_{2}\right), z\left(t_{2}\right)\right)-\sigma\left(c \mu^{\mathfrak{r}}\left(t_{2}\right), z\left(t_{1}\right)\right)\right\|$

$+\left\|\sigma\left(c \mu^{\mathfrak{r}}\left(t_{2}\right), z\left(t_{1}\right)\right)-\sigma\left(c \mu^{\mathfrak{r}}\left(t_{1}\right), z\left(t_{1}\right)\right)\right\|$

$\leqslant 2\left\|z\left(t_{2}\right)-z\left(t_{1}\right)\right\|+c\left\|\mu^{\mathfrak{r}}\left(t_{2}\right)-\mu^{\mathfrak{r}}\left(t_{1}\right)\right\| \leqslant 2 h_{1}+c h_{2}<k$

It follows that $\kappa_{2}$ is uniformly continuous over $\mathbb{R}_{\geqslant}$, being the product of uniformly continuous functions over $\mathbb{R}_{\geqslant}$.

Since $x, \xi \in L^{\infty}\left(\mathbb{R}_{\geqslant}, \mathbb{R}^{n}\right)$ and by claim $\# 1$, the $\Omega$-limit set $\Omega_{*}$ of the trajectory $(x(t), \xi(t), \mu(t))$ of $(26)-(7)-(9)$ is nonempty, compact and invariant and by virtue of claim $\# 2$ it is contained in the set $\mathfrak{R}:=\{(x, \xi, \mu): C x+\psi(x)=$ $\left.C \xi+\psi\left(\sigma\left(c \mu^{\mathfrak{r}}, \xi\right)\right), \sigma\left(c \mu^{\mathfrak{r}}, \xi\right)=\xi\right\}$. But any trajectory $\left(x_{*}(t), \xi_{*}(t), \mu_{*}(t)\right)$ of $(26)-(7)-(9)$ contained in $\Re$ must satisfy the equations $\dot{x}_{*}(t) \equiv A x_{*}(t)+\phi\left(x_{*}(t)\right), \dot{\xi}_{*}(t)=$ $A \xi_{*}(t)+\phi\left(\xi_{*}(t)\right)$ and $C x_{*}(t)+\psi\left(x_{*}(t)\right) \equiv C \xi_{*}(t)+$ $\psi\left(\xi_{*}(t)\right)$, which by assumption $H 2$ implies that $x_{*}(t) \equiv$ $\xi_{*}(t)$. Therefore, by attractiveness and invariance of $\Omega_{*}$, $\lim _{t \rightarrow \infty}\|x(t)-\xi(t)\|=0$ which proves the proposition.

\subsection{The predictor for $x(t)$}

The vector of the initial conditions $x_{0}, \hat{\mu}, \hat{\xi}$ will be denoted in what follows by $\varphi_{0}$ and recall that $v^{(j)}(t):=$ 
$\mu^{(0)}\left(t+j \frac{\Delta}{m}\right)$ and $y^{(j)}(t):=y\left(t+j \frac{\Delta}{m}\right)$ for $j=0, \ldots, m$. We will prove that $\xi^{(j)}(t)$ and $\mu^{(j)}(t), j=1, \ldots, m$, are asymptotic estimates of $x^{(j)}(t)$ and, resp., $v^{(j)}(t)$ and, therefore, $\xi^{(m)}(t)$ is an asymptotic estimate of $x^{(m)}(t)=$ $x(t)$, which proves our main theorem 3 .

Proposition 7 Assume HO, H1 and $\mathrm{H} 2$ and let $c, \alpha, K>0, \Gamma, \Phi^{M}, \Psi^{M} \in \mathbb{R}^{n \times n}$ and the integer $m$ be as in section 5 . Then the trajectory $x\left(\cdot, x_{0}\right)$, $\xi^{(j)}\left(\cdot, \varphi_{0}\right), \mu^{(j)}\left(\cdot, \varphi_{0}\right), j=1, \ldots, m$, of (7)-(9)-(11)-(12)(16)-(17) is defined and bounded for all times and initial conditions $\varphi_{0}$ and

$$
\begin{aligned}
& \lim _{t \rightarrow+\infty}\left(x^{(j)}\left(t, x_{0}\right)-\xi^{(j)}\left(t, \varphi_{0}\right)\right)=0 \\
& \lim _{t \rightarrow+\infty}\left(v^{(j)}\left(t, \varphi_{0}\right)-\mu^{(j)}\left(t, \varphi_{0}\right)\right)=0, j=1, \ldots, m .
\end{aligned}
$$

PROOF. Throughout the proof we will omit the dependence of the trajectories from the initial conditions. First of all, $\mu^{(j)}(t), j=1, \ldots, m$, satisfies for all times $t$ in its maximum right extension domain

$$
\mu^{(j)}(t)=\mu^{(j-1)}(t)+\int_{t-\frac{\Delta}{m}}^{t} G\left(\mu^{(j)}(s), \xi^{(j)}(s)\right) d s
$$

Indeed, by time-differentiating (50) we obtain (17) and the result follows from uniqueness of trajectories.

In a similar way we show that $\xi^{(j)}(t), j=1, \ldots, m$, satisfies for all times $t$ in its maximum right extension domain

$$
\xi^{(j)}(t)=\xi^{(j-1)}(t)+\int_{t-\frac{\Delta}{m}}^{t} M\left(\mu^{(j)}(s), \xi^{(j)}(s)\right) d s
$$

On the other hand, by definition $\gamma^{(j)}(t)$ and $x^{(j)}(t), j=$ $1, \ldots, m$, (defined and bounded for all times by $H 1$ and claim \#1 of proposition 6) satisfy for all $t \geqslant 0$

$$
\begin{aligned}
v^{(j)}(t) & =v^{(j-1)}(t)+\int_{t-\frac{\Delta}{m}}^{t}\left[G\left(v^{(j)}(s), \xi^{(0)}\left(s+j \frac{\Delta}{m}\right)\right)\right. \\
& \left.+Q\left(v^{(j)}(s), \xi^{(0)}\left(s+j \frac{\Delta}{m}\right), y^{(j)}(s)\right)\right] d s, \\
x^{(j)}(t) & =x^{(j-1)}(t)+\int_{t-\frac{\Delta}{m}}^{t}\left[A x^{(j)}(s)+\phi\left(x^{(j)}(s)\right)\right] d s
\end{aligned}
$$

Set

$$
e^{(j)}:=x^{(j)}-\xi^{(j)}, \omega^{(j)}:=v^{(j)}-\mu^{(j)}, j=1, \ldots, m .
$$

The proof of the proposition proceeds by induction. First, we prove the boundedness of $e^{(j)}(t)$ and $\omega^{(j)}(t)$ for all $j=1, \ldots, m$ and, finally, using invariance theorems we prove their convergence to zero.
Boundedness of $e^{(j)}(t)$ and $\omega^{(j)}(t), j=1, \ldots, m$. In view of the boundedness of $v^{(j)}(t)$ (proposition 6) it is sufficient to prove the boundedness of $e^{(j)}(t)$ and $\mu^{(j)}(t)$, $j=1, \ldots, m$. Fix $j \in\{1, \ldots, m-1\}$ and assume that

$$
\begin{aligned}
1 \leqslant \mu^{(j-1)}(t) & \leqslant \mathfrak{Z}^{(j-1)} \\
\left\|e^{(j-1)}(t)\right\| & \leqslant \mathfrak{Z}^{(j-1)}
\end{aligned}
$$

for all $t \geqslant 0$ and for some $\mathfrak{Z}^{(j-1)}>0$ (depending on the initial condition $\varphi_{0}$ ). First of all, notice that, by (50) and the induction hypothesis (54) and since $G$ is non-negative, $\mu^{(j)}(t) \geqslant 1$ for all $t \geqslant 0$. From (50) and the induction hypothesis (54), since $\mu^{1-2\left|\min _{i} \mathfrak{g}_{i}\right|} \| \mu^{-\mathfrak{r}} \diamond$ $\sigma\left(c \mu^{\mathfrak{r}}, w\right) \|^{2} \leqslant \mu n^{2} c^{2}$ for all $\mu \geqslant 1$ and $w \in \mathbb{R}^{n}$,

$$
\begin{aligned}
\mu^{(j)}(t) & =\mu^{(j-1)}(t)+\int_{t-\frac{\Delta}{m}}^{t} G\left(\mu^{(j)}(s), \xi^{(j)}(s)\right) d s \\
& \leqslant \mathfrak{Z}^{(j-1)}+n^{2} c^{2} \int_{t-\frac{\Delta}{m}}^{t} \mu^{(j)}(s) d s
\end{aligned}
$$

It follows, on account of the choice of $m$ in (23) and by (ii) of lemma 8 with $s:=\mu^{(j)}, k_{0}:=n^{2} c^{2}, k_{1}:=\mathfrak{Z}^{(j-1)}$ and $\delta:=\frac{\Delta}{m}$, that

$\mu^{(j)}(t) \leqslant \tilde{\mathfrak{Z}}^{(j)}$

for all $t \geqslant 0$ and for $\tilde{\mathfrak{Z}}^{(j)}>0$ (depending on $\left.\varphi_{0}\right)$.

Also, let $\beta>0$ be (depending on $\varphi_{0}$ ) such that

$\left\|x^{(j)}(t)\right\| \leqslant \beta$

for all $t \geqslant 0$ (assumption H1). Moreover, on account of part (iv) of lemma $11 \phi$ is i.h.u.b. with quadruple $(\mathfrak{r}, \mathfrak{g}+\mathfrak{r}, \mathfrak{g}, \Phi)$, i.e. for all $\mu \geqslant 1$ and $w, z$

$$
\begin{aligned}
& \left\langle\left\langle\phi\left(\mu^{\mathfrak{r}} \diamond w\right)-\phi\left(\mu^{\mathfrak{r}} \diamond z\right)\right\rangle\right. \\
& \leq \mu^{\mathfrak{g}+\mathfrak{r}} \diamond\left(\Phi(w, z)\left\langle\left\langle\mu^{\mathfrak{g}} \diamond(w-z)\right\rangle\right)\right.
\end{aligned}
$$

From this with $\mu:=1$ and $w:=x^{(j)}$ and $z:=$ $\sigma^{c\left(\mu^{(j)}\right)^{\mathrm{r}}}\left(x^{(j)}\right)$, using (58) and (ii) of lemma 9, we obtain for all $t \geqslant 0$

$$
\begin{aligned}
& \left\|\phi\left(x^{(j)}(t)\right)-\phi\left(\sigma^{c\left(\mu^{(j)}\right)^{\mathfrak{r}}(t)}\left(x^{(j)}(t)\right)\right)\right\| \\
& \leqslant \max _{\|w\|,\|z\| \leqslant \beta}\|\Phi(w, z)\langle(w-z\rangle\rangle\|:=\alpha
\end{aligned}
$$

for $\alpha>0$ (depending on $\varphi_{0}$ ).

On the other hand, from (59) with $\mu:=\mu^{(j)}, w:=$ $\left(\mu^{(j)}\right)^{-\mathfrak{r}} \diamond \sigma^{c\left(\mu^{(j)}\right)^{\mathfrak{r}}}\left(x^{(j)}\right)$ and $z:=\left(\mu^{(j)}\right)^{-\mathfrak{r}} \diamond \sigma^{c\left(\mu^{(j)}\right)^{\mathfrak{r}}}\left(\xi^{(j)}\right)$, using (i) of lemma 9 and since $\left\|\mu^{-\mathfrak{r}} \diamond \sigma\left(c \mu^{\mathfrak{r}}, w\right)\right\| \leqslant n c$ 
and $\mu^{\mathfrak{v}} \leq \mu^{\langle\langle\mathfrak{v}\rangle}$ for all $\mu \geqslant 1, \mathfrak{v} \in \mathbb{R}^{n}$ and $w \in \mathbb{R}^{n}$, we obtain for all $t \geqslant 0$

$\left\|\phi\left(\sigma^{c\left(\mu^{(j)}\right)^{\mathfrak{r}}(t)}\left(x^{(j)}(t)\right)\right)-\phi\left(\sigma^{c\left(\mu^{(j)}\right)^{\mathfrak{r}}(t)}\left(\xi^{(j)}(t)\right)\right)\right\| \leq 2$.

$\max _{\|w\|,\|z\| \leqslant c n}\left\|\mu^{\langle\langle\mathfrak{r}+\mathfrak{g}\rangle\rangle}(t) \diamond\left(\Phi(w, z)\left\langle\left\langle\mu^{\langle\langle\mathfrak{g}\rangle\rangle}(t) \diamond(w-z)\right\rangle\right\rangle\right)\right\|$

$1 \leqslant \mu \leqslant \mu(j)(t)$

$:=\kappa\left(\mu^{(j)}(t)\right)$

with increasing continuous non-negative function $\kappa$.

Subtracting (51) from (53) and taking into account the monotonicity of $\mathrm{k}$ with (57) and the induction hypothesis (55) together with (60) and (61), we have for all $t \geqslant 0$

$\left\|e^{(j)}(t)\right\| \leqslant \mathfrak{Z}^{(j-1)}+\frac{\Delta}{m}\left[\alpha+\kappa\left(\tilde{\mathfrak{Z}}^{(j)}\right)\right]+\|A\| \int_{t-\frac{\Delta}{m}}^{t}\left\|e^{(j)}(s)\right\| d s$

On account of (23), (57) and applying (i) of lemma 8 with $s:=\left\|e^{(j)}\right\|, k_{0}:=\|A\|, k_{1}:=\mathfrak{Z}^{(j-1)}+\frac{\Delta}{m}\left[\alpha+\kappa\left(\tilde{\mathfrak{Z}}^{(j)}\right)\right]$ and $\delta:=\frac{\Delta}{m}$, it follows that

$\left\|e^{(j)}(t)\right\| \leqslant \mathfrak{Z}^{(j)}, 1 \leqslant \mu^{(j)}(t) \leqslant \mathfrak{Z}^{(j)}$

for all $t \geqslant 0$ and for some $\mathfrak{Z}^{(j)} \geqslant \tilde{\mathfrak{Z}}^{(j)}$. This proves (by induction) the boundedness of $e^{(j)}(t)$ and $\mu^{(j)}(t)$ (and therefore $\left.\boldsymbol{\omega}^{(j)}(t)\right), j=1, \ldots, m$.

Convergence of $e^{(j)}(t)$ and $\omega^{(j)}(t), j=1, \ldots, m$, to zero. We proceed again by induction. Assume that for some $j \in\{1, \ldots, m-1\}$

$\lim _{t \rightarrow+\infty} e^{(i)}(t)=0, \lim _{t \rightarrow+\infty} \omega^{(i)}(t)=0, i=0, \ldots, j-1$.

We have established above that $\left(e^{(i)}(t), \omega^{(i)}(t)\right)$ are bounded for all $i=1, \ldots, j$. It follows that the $\Omega$-limit set $\Omega_{*}$ of each trajectory $\left(x^{(j)}(t), \xi^{(j)}(t), v^{(j)}(t), \mu^{(j)}(t)\right)$ is non-empty, compact and invariant (corollary 5.6 of Smith (2011)). But $\lim _{t \rightarrow+\infty}\left[x^{(0)}(t)-\xi^{(0)}(t)\right]=0$ (proposition 6) and since by definition $x^{(j)}(t) \equiv$ $x^{(0)}\left(t+j \frac{\Delta}{m}\right)$

$\lim _{t \rightarrow+\infty}\left[x^{(j)}(t)-\xi^{(0)}\left(t+j \frac{\Delta}{m}\right)\right]=0$

Moreover, recalling claim \#2 (proof of proposition 6) with $t+j \frac{\Delta}{m}$ replacing $t$

$$
\begin{aligned}
& \left.\lim _{\substack{t \rightarrow+\infty \\
=0}}\left[-\xi^{(0)}\left(t+j \frac{\Delta}{m}\right)+\sigma^{c\left(\mu^{(0)}\right)^{\mathfrak{r}}\left(t+j \frac{\Delta}{m}\right)}\left(\xi^{(0)}\left(t+j \frac{\Delta}{m}\right)\right)\right)\right] \\
&
\end{aligned}
$$

Therefore, by (i) of lemma 9 and since by definition $v^{(j)}(t) \equiv \mu^{(0)}\left(t+j \frac{\Delta}{m}\right)$

$\left.\lim _{t \rightarrow+\infty}\left[\sigma^{c\left(v^{(j)}\right)^{\mathfrak{r}}(t)}\left(x^{(j)}(t)\right)\right)-x^{(j)}(t)\right]$

$\left.\left.=\lim _{t \rightarrow+\infty}\left[\sigma^{c\left(v^{(j)}\right)^{\mathfrak{r}}(t)}\left(x^{(j)}(t)\right)\right)-\sigma^{c\left(v^{(j)}\right)^{\mathfrak{r}}(t)}\left(\xi^{(0)}\left(t+j \frac{\Delta}{m}\right)\right)\right)\right]$

$+\lim _{t \rightarrow+\infty}\left[-x^{(j)}(t)+\xi^{(0)}\left(t+j \frac{\Delta}{m}\right)\right]+\lim _{t \rightarrow+\infty}\left[-\xi^{(0)}\left(t+j \frac{\Delta}{m}\right)\right.$

$\left.\left.+\sigma^{c\left(\mu^{(0)}\right)^{\mathfrak{r}}\left(t+j \frac{\Delta}{m}\right)}\left(\xi^{(0)}\left(t+j \frac{\Delta}{m}\right)\right)\right)\right]=0$

and

$$
\begin{aligned}
& \lim _{t \rightarrow+\infty} Q\left(v^{(j)}(t), \xi^{(0)}\left(t+j \frac{\Delta}{m}\right), y^{(j)}(t)\right)=0 \\
& \lim _{t \rightarrow+\infty} G\left(v^{(j)}(t), \xi^{(0)}\left(t+j \frac{\Delta}{m}\right)\right)=0
\end{aligned}
$$

It follows from (64), (65), (66), invariance of $\Omega_{*}$ and the induction hypothesis (63) that each trajectory $\left(x_{*}^{(j)}(t)\right.$, $\left.\xi_{*}^{(j)}(t), v_{*}^{(j)}(t), \mu_{*}^{(j)}(t)\right)$ (with initial condition $\varphi_{*}$ ) contained in $\Omega_{*}$ satisfies (50)-(51)-(52) and (53) for all $t \geqslant 0$ with

$$
\begin{aligned}
& x_{*}^{(j-1)}(t) \equiv \xi_{*}^{(j-1)}(t), v_{*}^{(j-1)}(t) \equiv \mu_{*}^{(j-1)}(t) \\
& \sigma^{c\left(v_{*}^{(j)}\right)^{\mathfrak{r}}(t)}\left(x_{*}^{(j)}(t)\right) \equiv x_{*}^{(j)}(t), x_{*}^{(j)}(t) \equiv \xi_{*}^{(0)}\left(t+j \frac{\Delta}{m}\right), \\
& Q\left(v_{*}^{(j)}(t), \xi_{*}^{(0)}\left(t+j \frac{\Delta}{m}\right), y_{*}^{(j)}(t)\right) \equiv 0 \\
& G\left(v_{*}^{(j)}(t), \xi_{*}^{(0)}\left(t+j \frac{\Delta}{m}\right)\right) \equiv 0
\end{aligned}
$$

where $y_{*}^{(j)}$ is the output trajectory $y^{(j)}$ which corresponds to $x_{*}^{(j)}$. If we show that $e_{*}^{(j)}(t):=x_{*}^{(j)}(t)-\xi_{*}^{(j)}(t)$ and $\omega_{*}^{(j)}(t):=v_{*}^{(j)}(t)-\mu_{*}^{(j)}(t)$ are zero for all $t \geqslant \frac{\Delta}{m}$ then, by attractiveness and invariance of $\Omega_{*}$, $\lim _{t \rightarrow+\infty} e^{(j)}(t)=0$ and $\lim _{t \rightarrow+\infty} \omega^{(j)}(t)=0$ and this concludes the proof of (49) by induction.

Let us prove that $\omega_{*}^{(j)}(t)$ and $e_{*}^{(j)}(t)$ are zero for all $t \geqslant$ $\frac{\Delta}{m}$. Using (50)-(51)-(52) and (53) with the additional constraints (67), we have for all $t \geqslant 0$

$$
\begin{aligned}
e_{*}^{(j)}(t) & =\int_{t-\frac{\Delta}{m}}^{t}\left[A \hat{e}_{*}^{(j)}(s)+\phi\left(\sigma^{c\left(v_{*}^{(j)}\right)^{\mathfrak{r}}(s)}\left(x_{*}^{(j)}(s)\right)\right)\right. \\
& \left.-\phi\left(\sigma^{c\left(\mu_{*}^{(j)}\right)^{\mathfrak{r}}(s)}\left(\xi_{*}^{(j)}(s)\right)\right)\right] d s \\
\omega_{*}^{(j)}(t) & =-\int_{t-\frac{\Delta}{m}}^{t} G\left(\mu_{*}^{(j)}(s), \xi_{*}^{(j)}(s)\right) d s
\end{aligned}
$$

By (i) of lemma 9 and lemma 10 and using the triangle inequality, $\left\|\sigma^{h}(w)-\sigma^{k}(z)\right\| \leqslant\|h-k\|+2\|w-z\|$ for all $w, z \in \mathbb{R}^{n}$ and $h, k \in \mathbb{R}_{>}^{n}$. Using this with (iii) of 
lemma 9 and (59) with $\mu:=1, w:=\sigma^{c\left(v_{*}^{(j)}\right)^{\mathfrak{r}}}\left(x_{*}^{(j)}\right)$ and $z:=\sigma^{c\left(\mu_{*}^{(j)}\right)^{\mathfrak{r}}}\left(\xi_{*}^{(j)}\right)$ together with the boundedness of $v_{*}^{(j)}$ and $\mu_{*}^{(j)}$,

$$
\begin{aligned}
& \left\|\phi\left(\sigma^{c\left(v_{*}^{(j)}\right)^{\mathfrak{r}}(t)}\left(x_{*}^{(j)}(t)\right)\right)-\phi\left(\sigma^{c\left(\mu_{*}^{(j)}\right)^{\mathfrak{r}}(t)}\left(\xi_{*}^{(j)}(t)\right)\right)\right\| \quad(70) \\
& \leqslant\left(\max _{\substack{\langle w\rangle \leq c\left(v_{*}^{(j)}\right) \mathfrak{r}(t) \\
*\langle z\rangle \leq c\left(\mu_{*}^{(j)}\right)^{\mathfrak{r}}(t)}}\|\Phi(w, z)\|\right)\left[c\left\|\left(v_{*}^{(j)}\right)^{\mathfrak{r}}(t)-\left(\mu_{*}^{(j)}\right)^{\mathfrak{r}}(t)\right\|\right. \\
& \left.+2\left\|e_{*}^{(j)}(t)\right\|\right] \leqslant \rho_{1}\left[\left\|\left(v_{*}^{(j)}\right)^{\mathfrak{r}}(t)-\left(\mu_{*}^{(j)}\right)^{\mathfrak{r}}(t)\right\|+\left\|e_{*}^{(j)}(t)\right\|\right]
\end{aligned}
$$

for all $t \geqslant 0$ and for some $\rho_{1}>0$ (depending on the initial condition $\left.\varphi_{*}\right)$. But the function $\mu \mapsto \mu^{q}, q>0$, is continuous over any closed interval $\left[1, \mu_{\infty}\right]$ and with bounded derivative over $\left(1, \mu_{\infty}\right)$. By the mean value theorem $\left|\mu^{q}-\nu^{q}\right| \leqslant q\left(\sup _{\mu \in\left(1, \mu_{\infty}\right)} \mu^{q-1}\right)|\mu-v|$ for all $\mu, \nu \in$ $\left[1, \mu_{\infty}\right]$. Therefore, by boundedness of $\mu_{*}^{(j)}(t)$ and $v_{*}^{(j)}(t)$, $\left\|\left(v_{*}^{(j)}\right)^{\mathfrak{r}}(t)-\left(\mu_{*}^{(j)}\right)^{\mathfrak{r}}(t)\right\| \leqslant \rho_{2}\left|\omega_{*}^{(j)}(t)\right|$ for all $t \geqslant 0$ and for some $\rho_{2}>0$ (depending on $\varphi_{*}$ ). From (68) with (70) we get for all $t \geqslant 0$

$$
\left\|e_{*}^{(j)}(t)\right\| \leqslant\left[\|A\|+\rho_{1}\left(1+\rho_{2}\right)\right] \int_{t-\frac{\Delta}{m}}^{t}\left[\left|\omega_{*}^{(j)}(t)\right|+\left\|e_{*}^{(j)}(t)\right\|\right] d s
$$

On the other hand, on account of $\sigma^{c\left(v_{*}^{(j)}\right)^{\mathfrak{r}}(t)}\left(x_{*}^{(j)}(t)\right) \equiv$ $x_{*}^{(j)}(t)$ in (67), by (i) of lemma 9 , lemma 10 and using the triangle inequality with $\left\|\left(v_{*}^{(j)}\right)^{\mathfrak{r}}(t)-\left(\mu_{*}^{(j)}\right)^{\mathfrak{r}}(t)\right\| \leqslant$ $\rho_{2}\left|\omega_{*}^{(j)}(t)\right|$ for all $t \geqslant 0$, we get for all $t \geqslant 0$

$$
\begin{aligned}
& \left|G\left(\mu_{*}^{(j)}(t), \xi_{*}^{(j)}(t)\right)\right| \\
& \leqslant \rho_{3}\left\|\sigma\left(c\left(\mu_{*}^{(j)}\right)^{\mathfrak{r}}(t), \xi_{*}^{(j)}(t)-\sigma\left(c\left(\mu_{*}^{(j)}\right)^{\mathfrak{r}}(t), \xi_{*}^{(j)}(t)\right)\right)\right\| \\
& \leqslant \rho_{3}\left[\left\|e_{*}^{(j)}(t)\right\|+\| \sigma\left(c\left(\mu_{*}^{(j)}\right)^{\mathfrak{r}}(t), x_{*}^{(j)}(t)\right)\right. \\
& -\sigma\left(c\left(v_{*}^{(j)}\right)^{\mathfrak{r}}(t), \xi_{*}^{(j)}(t)\right) \| \leqslant \rho_{3}\left[3\left\|e_{*}^{(j)}(t)\right\|+\rho_{2}\left|\omega_{*}^{(j)}(t)\right|\right]
\end{aligned}
$$

for some $\rho_{3}>0$ (depending on the initial condition $\varphi_{*}$ ) and therefore from (69)

$\left|\omega_{*}^{(j)}(t)\right| \leqslant \rho_{3}\left(3+\rho_{2}\right) \int_{t-\frac{\Delta}{m}}^{t}\left[\left|\omega_{*}^{(j)}(t)\right|+\left\|e_{*}^{(j)}(t)\right\|\right] d s$

Summing (71) and (72), applying part (ii) of lemma 8 with $s:=\left|\omega_{*}^{(j)}\right|+\left\|e_{*}^{(j)}\right\|, k_{0}:=\|A\|+\rho_{1}\left(1+\rho_{2}\right)+\rho_{3}(3+$ $\left.\rho_{2}\right), k_{1}:=0$ and $\delta:=\frac{\Delta}{m}$, we conclude that $\omega_{*}^{(j)}(t)=0$ and $e_{*}^{(j)}(t)=0$ for all $t \geqslant \frac{\Delta}{m}$. This concludes the proof.

\section{Conclusions}

We have presented a class of nonlinear predictors for stable systems with delayed measurements and constant and known delay. These nonlinear predictors consist of $m+1$ couple of filters, each couple generates an estimate of the state vector (and its maximum magnitude) at some delayed time instant which differ from the previous by a small fraction of the overall delay. This fraction is sufficiently small to guarantee convergence of the estimate to the real value. These estimates are used by consecutive couples of filters to generate the estimates at the next delayed time instant, with the last couple generating the desired prediction of the state. Further research will be devoted to the case of unknown delay and unstable systems.

\section{Acknowldegments}

The author wishes to thank anonymous reviewers for many precise and fruitful remarks which have finally led to a remarkably improved version of the paper.

\section{A Incremental homogeneity in the generalized sense: a review}

The notion of (incremental) homogeneity in the generalized sense has been introduced in Battilotti (2011) (see also Battilotti (2013)) in the context of semi-global stabilization and observer design problems. Here we recall this notion in a slightly more general form.

\section{A.1 Definitions}

$A$ function $\phi \in C^{0}\left(\mathbb{R}_{>} \times \mathbb{R}^{n}, \mathbb{R}^{l}\right),(\mu, x) \mapsto \phi(\mu, x)$, is said to be incrementally homogeneous in the generalized sense (i.h.) with quadruple $(\mathfrak{r}, \mathfrak{d}, \mathfrak{h}, \Phi)$ if there exist $\mathfrak{d} \in$ $\mathbb{R}^{l}, \mathfrak{h} \in \mathbb{R}^{n}, \mathfrak{r} \in \mathbb{R}_{>}^{n}$ and $\Phi \in C^{0}\left(\mathbb{R}^{2 n}, \mathbb{R}^{l \times n}\right)$ such that for all $\varepsilon>0$ and $w, z \in \mathbb{R}^{n}$

$$
\phi\left(\varepsilon, \varepsilon^{\mathfrak{r}} \diamond w\right)-\phi\left(\varepsilon, \varepsilon^{\mathfrak{r}} \diamond z\right)=\varepsilon^{\mathfrak{d}} \diamond\left(\Phi(w, z)\left(\varepsilon^{\mathfrak{h}} \diamond(w-z)\right)\right)
$$

Note that the function $\phi$ may depend on the dilating parameter itself. The function $x \mapsto \phi(x):=x_{1}+x_{2}^{3}(\phi$ does not depend on the dilating parameter) is i.h. with quadruple $(\mathfrak{r}, 0, \mathfrak{h}, \Phi)$, where $\mathfrak{r}:=(1,2)^{T}, \mathfrak{h}:=(1,6)^{T}$ and $\Phi(w, z):=\left(1, w_{2}^{2}+z_{2}^{2}+z_{2} w_{2}\right)$. The function $(\mu, x) \mapsto$ $\phi(\mu, x):=\mu\left(x_{1}+x_{2}^{3}\right)$ ( $\phi$ does depend on the dilating parameter) is i.h. with quadruple $(\mathfrak{r}, 1, \mathfrak{h}, \Phi)$.

When restricting to $z=0$ in the above definition, we obtain the definition of homogeneity in the generalized sense. For functions $x \mapsto \phi(x)$ this definition generalizes the classical notion of homogeneity (Rosier (1992)). Note that homogeneity in the generalized sense is characterized by two vector degrees $(\mathfrak{d}, \mathfrak{h})$ instead of being characterized by only one degree $\mathfrak{d}$ as in the case of homogeneity in the classical sense and the function may depend on the dilating parameter itself.

There are functions, like $\sin x$, which are not i.h. but their absolute value is bounded by the absolute value of 


\section{CONFIDENTIAL. Limited circulation. For review only Automatica submission 13-0695.3}

a function which is i.h. This motivates the following definition $(\langle\langle a\rangle\rangle$ denotes the column vector of the absolute values of the elements of $\left.a \in \mathbb{R}^{n}\right)$.

A function $\phi \in C^{0}\left(\mathbb{R}_{>} \times \mathbb{R}^{n}, \mathbb{R}^{l}\right),(\mu, x) \mapsto \phi(\mu, x)$, is said to be incrementally homogeneous in the upper bound in the generalized sense (i.h.u.b.) with quadruple $(\mathfrak{r}, \mathfrak{d}, \mathfrak{h}, \Phi)$ if there exist $\mathfrak{d} \in \mathbb{R}^{l}, \mathfrak{h} \in \mathbb{R}^{n}, \mathfrak{r} \in \mathbb{R}_{>}^{n}$, $\Phi \in C^{0}\left(\mathbb{R}^{2 n}, \mathbb{R}_{\geqslant}^{l \times n}\right)$ such that for all $\varepsilon \geqslant 1$ and $w, z \in \mathbb{R}^{n}$

$\left\langle\left\langle\phi\left(\varepsilon, \varepsilon^{\mathfrak{r}} \diamond w\right)-\phi\left(\varepsilon, \varepsilon^{\mathfrak{r}} \diamond z\right)\right\rangle \leq \varepsilon^{\mathfrak{o}} \diamond\left(\Phi(w, z)\left\langle\left\langle\varepsilon^{\mathfrak{h}} \diamond(w-z)\right\rangle\right)\right.\right.$

The function $(\mu, x) \mapsto \phi(\mu, x):=\mu\left(x_{2} \quad x_{2}^{3} g\left(x_{1}\right)\right)^{T}, g$ any bounded and globally Lipschitz continuous function, is i.h.u.b. with triple $(\mathfrak{r}, \mathfrak{d}, \mathfrak{h}, \Phi)$, where $\mathfrak{r}:=(1,2)^{T}, \mathfrak{d}:=$ $(3,7)^{T}, \mathfrak{h}:=(1,0)^{T}$ and

$\Phi(w, z):=\left(\begin{array}{cc}0 & 1 \\ z_{2}^{3} \frac{\left|g\left(w_{1}\right)-g\left(z_{1}\right)\right|}{\left|w_{1}-z_{1}\right|} & \left|w_{2}^{2}+z_{2}^{2}+w_{2} z_{2}\right|\left|g\left(w_{1}\right)\right|\end{array}\right)$

(here we used $w_{2}^{3} g\left(w_{1}\right)-z_{2}^{3} g\left(z_{1}\right)=\left(w_{2}^{3}-z_{2}^{3}\right) g\left(w_{1}\right)+$ $\left.z_{2}^{3}\left(g\left(w_{1}\right)-g\left(z_{1}\right)\right)\right)$.

\section{A.2 Properties of incrementally homogeneous func-} tions

P0 (addition) For any i.h.u.b. (resp. i.h.) functions $\phi \in C^{0}\left(\mathbb{R}_{>} \times \mathbb{R}^{n}, \mathbb{R}^{l}\right),(\mu, y) \mapsto \phi(\mu, y)$, with quadruple $(\mathfrak{r}, \mathfrak{d}, \mathfrak{h}, \Phi)$ and $\psi \in C^{0}\left(\mathbb{R}_{>} \times \mathbb{R}^{n}, \mathbb{R}^{l}\right)$, $(\mu, y) \mapsto \psi(\mu, y)$, with quadruple $(\mathfrak{r}, \mathfrak{d}, \mathfrak{h}, \Psi)$, the function $(\mu, y) \mapsto \phi(\mu, y)+\psi(\mu, y)$ is i.h.u.b. (resp. i.h.) with quadruple $(\mathfrak{r}, \mathfrak{d}, \mathfrak{h}, \Phi+\Psi)$.

P1 Any i.h.u.b. (resp. i.h.) function $\phi \in C^{0}\left(\mathbb{R}_{>} \times \mathbb{R}^{n}, \mathbb{R}^{l}\right)$ with quadruple $(\mathfrak{r}, \mathfrak{d}, \mathfrak{h}, \Phi)$ and diagonal $\Phi$ is also i.h.u.b. (resp. i.h.) with quadruple $\left(\mathfrak{r}, \mathfrak{d}^{\prime}, \mathfrak{h}^{\prime}, \Phi\right)$ for all pairs $\left(\mathfrak{d}^{\prime}, \mathfrak{h}^{\prime}\right)$ such that $\mathfrak{d}+\mathfrak{h} \leq \mathfrak{d}^{\prime}+\mathfrak{h}^{\prime}\left(\right.$ resp. $\left.\mathfrak{d}+\mathfrak{h}=\mathfrak{d}^{\prime}+\mathfrak{h}^{\prime}\right)$.

In particular, we can replace the degrees $(\mathfrak{d}, \mathfrak{h})$ with some upper bounds $\left(\mathfrak{d}^{\prime}, \mathfrak{h}^{\prime}\right)$ or swap $:\left(\mathfrak{d}^{\prime}, \mathfrak{h}^{\prime}\right)=(\mathfrak{h}, \mathfrak{d})$.

P2 (composition) For any i.h.u.b. functions $\phi \in C^{0}\left(\mathbb{R}_{>} \times\right.$ $\left.\mathbb{R}^{s}, \mathbb{R}^{l}\right),(\mu, y) \mapsto \phi(\mu, y)$, with quadruple $(\mathfrak{r}, \mathfrak{d}, \mathfrak{h}, \Phi)$ and $\psi \in C^{0}\left(\mathbb{R}_{>} \times \mathbb{R}^{n}, \mathbb{R}^{s}\right),(\mu, x) \mapsto \psi(\mu, x)$, with quadruple $(\mathfrak{r},-\mathfrak{h}+\mathfrak{r}, \mathfrak{p}, \Psi)$ if there exists $\Phi^{M} \in C^{0}\left(\mathbb{R}^{2 s}, \mathbb{R}_{\geqslant}^{l \times s}\right)$ such that for all $\varepsilon \geqslant 1$ and $w, z \in \mathbb{R}^{n}$

$$
\Phi\left(\varepsilon^{-\mathfrak{r}} \diamond \psi\left(\varepsilon^{\mathfrak{r}} \diamond w\right), \varepsilon^{-\mathfrak{r}} \diamond \psi\left(\varepsilon^{\mathfrak{r}} \diamond z\right)\right) \leq \Phi^{M}(w, z)
$$

then $(\mu, x) \mapsto \phi(\mu, \psi(\mu, x))$ is i.h.u.b. with quadruple $\left(\mathfrak{r}, \mathfrak{d}, \mathfrak{p}, \Phi^{M} \Psi\right) .^{3}$

\footnotetext{
3 Using properties (2), for all $\varepsilon \geqslant 1$ and $w, z \in \mathbb{R}^{n}$

$\left\langle\left\langle\phi\left(\varepsilon, \psi\left(\varepsilon, \varepsilon^{\mathfrak{r}} \diamond w\right)\right)-\phi\left(\varepsilon, \psi\left(\varepsilon, \varepsilon^{\mathfrak{r}} \diamond z\right)\right)\right\rangle=\right.$
}

In particular, for $\phi$ with constant $\Phi$ (A.1) is trivially satisfied with $\Phi^{M}=\Phi$.

Let $(A, B)$ be in Brunowski canonical form. Note that $A^{T}$ is the Moore-Penrose pseudoinverse of $A$, viz. $A^{T} A A^{T}=$ $A^{T}, A A^{T} A=A,\left(A^{T} A\right)^{T}=A^{T} A$ and $\left(A A^{T}\right)^{T}=A A^{T}$. Therefore $I-A A^{T}$ is the orthogonal projection onto $(\operatorname{Im}\{A\})^{\perp}=\operatorname{Im}\left\{I-A A^{T}\right\}$ while $I-A^{T} A$ is the orthogonal projection onto $\left(\operatorname{Im}\left\{A^{T}\right\}\right)^{\perp}=\operatorname{Im}\left\{I-A^{T} A\right\}(\operatorname{Im}\{W\}$ denotes the vector space generated by the columns of the matrix $W$ ). It is easy to see that

P3.1 (shifting I) for any $\mathfrak{z} \in \operatorname{Im}\left\{I-A A^{T}\right\}$ (resp. $\left.\mathfrak{z} \in \operatorname{Im}\left\{I-A^{T} A\right\}\right)$ and i.h.u.b. (resp.i.h.) $\phi \in$ $C^{0}\left(\mathbb{R}_{>} \times \mathbb{R}^{n}, \mathbb{R}^{l}\right), \quad(\mu, x) \mapsto \phi(\mu, x)$, with quadruple $(\mathfrak{r}, \mathfrak{d}, \mathfrak{h}, \Phi)$, the function $(\mu, x) \mapsto A \phi(\mu, x)$ (resp. $\left.(\mu, x) \mapsto A^{T} \phi(\mu, x)\right)$ is i.h.u.b. (resp.i.h.) with quadruple $(\mathfrak{r}, A \mathfrak{d}+\mathfrak{z}, \mathfrak{h}, A \Phi)\left(\operatorname{resp} .\left(\mathfrak{r}, A^{T} \mathfrak{d}+\mathfrak{z}, \mathfrak{h}, A^{T} \Phi\right)\right) .{ }^{4}$

P3.2 (shifting II) for any $\mathfrak{z} \in \operatorname{Im}\left\{I-A^{T} A\right\}$ (resp. $\mathfrak{z} \in$ $\left.\operatorname{Im}\left\{I-A A^{T}\right\}\right)$ and i.h.u.b. (resp.i.h.) $\phi \in C^{0}\left(\mathbb{R}_{>} \times\right.$ $\left.\mathbb{R}^{n}, \mathbb{R}^{l}\right),(\mu, x) \mapsto \phi(\mu, x)$, with quadruple $(\mathfrak{r}, \mathfrak{d}, \mathfrak{h}, \Phi)$ and constant $\Phi$, the function $(\mu, x) \mapsto \phi(\mu, A x)$ (resp. $\left.(\mu, x) \mapsto \phi\left(\mu, A^{T} x\right)\right)$ is i.h.u.b. (resp.i.h.) with quadruple $\left(\mathfrak{r}, \mathfrak{d}, A^{T}(\mathfrak{h}-\mathfrak{r})+\mathfrak{r}+\mathfrak{z}, \Phi A\right)($ resp. $(\mathfrak{r}, \mathfrak{d}, A(\mathfrak{h}-\mathfrak{r})+$ $\left.\left.\mathfrak{r}+\mathfrak{z}, \Phi A^{T}\right)\right)^{5}$

$$
\begin{aligned}
& \left\langle\left\langle\phi\left(\varepsilon, \varepsilon^{\mathfrak{r}} \diamond \varepsilon^{-\mathfrak{r}} \diamond \psi\left(\varepsilon, \varepsilon^{\mathfrak{r}} \diamond w\right)\right)-\phi\left(\varepsilon, \varepsilon^{\mathfrak{r}} \diamond \varepsilon^{-\mathfrak{r}} \diamond \psi\left(\varepsilon, \varepsilon^{\mathfrak{r}} \diamond z\right)\right)\right\rangle\right.
\end{aligned}
$$

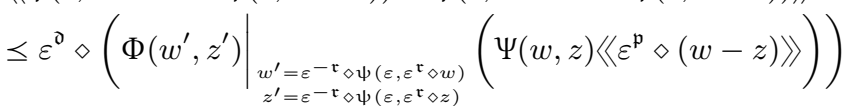

$$
\begin{aligned}
& \leq \varepsilon^{\mathfrak{d}} \diamond\left(\left(\Phi^{M}(w, z) \Psi(w, z)\right)\left\langle\left\langle\varepsilon^{\mathfrak{p}} \diamond(w-z)\right\rangle\right)\right)
\end{aligned}
$$

4 Using $A \varepsilon^{\mathfrak{d}}=A A^{T} A \varepsilon^{\mathfrak{d}}=A A^{T} \varepsilon^{A \mathfrak{d}+\mathfrak{z}}$ with $\mathfrak{z} \in \operatorname{Im}\left\{I-A A^{T}\right\}$, for all $\varepsilon \geqslant 1$ and $w, z \in \mathbb{R}^{n}$

$$
\begin{aligned}
& \left\langle\left\langle A \phi\left(\varepsilon, \varepsilon^{\mathfrak{r}} \diamond w\right)-A \phi\left(\varepsilon, \varepsilon^{\mathfrak{r}} \diamond z\right)\right\rangle\right\rangle \\
& \leq\left(A \varepsilon^{\mathfrak{o}}\right) \diamond\left(A\left(\Phi(w, z)\left\langle\left\langle\varepsilon^{\mathfrak{h}} \diamond(w-z)\right\rangle\right)\right)\right. \\
& =\varepsilon^{A \mathfrak{d}+\mathfrak{z}} \diamond\left(A \Phi(w, z)\left\langle\left\langle\varepsilon^{\mathfrak{h}} \diamond(w-z)\right\rangle\right)\right)
\end{aligned}
$$

${ }^{5}$ Using $A A^{T} \varepsilon^{\mathfrak{h}-\mathfrak{r}}=A \varepsilon^{A^{T}(\mathfrak{h}-\mathfrak{r})+\mathfrak{z}}$ with $\mathfrak{z} \in \operatorname{Im}\left\{I-A A^{T}\right\}$ and properties (2) and (3), for all $\varepsilon \geqslant 1$ and $w, z \in \mathbb{R}^{n}$

$$
\begin{aligned}
& \left\langle\left\langle\phi\left(\varepsilon, A\left(\varepsilon^{\mathfrak{r}} \diamond w\right)\right)-\phi\left(\varepsilon, A\left(\varepsilon^{\mathfrak{r}} \diamond z\right)\right)\right\rangle\right. \\
& =\left\langle\left\langle\phi\left(\varepsilon, \varepsilon^{\mathfrak{r}} \diamond \varepsilon^{-\mathfrak{r}} \diamond\left(A\left(\varepsilon^{\mathfrak{r}} \diamond w\right)\right)\right)-\phi\left(\varepsilon, \varepsilon^{\mathfrak{r}} \diamond \varepsilon^{-\mathfrak{r}} \diamond\left(A\left(\varepsilon^{\mathfrak{r}} \diamond z\right)\right)\right)\right\rangle\right. \\
& \leq \varepsilon^{\mathfrak{o}} \diamond\left(\Phi \left\langle\left\langle\varepsilon^{\mathfrak{h}-\mathfrak{r}} \diamond\left(A\left(\varepsilon^{\mathfrak{r}} \diamond\langle\langle w-z\rangle)\right)\right)\right.\right.\right. \\
& \leq \varepsilon^{\mathfrak{o}} \diamond\left(\Phi \left\langle\left\langle\left(A A^{T} \varepsilon^{\mathfrak{h}-\mathfrak{r}}\right) \diamond\left(A\left(\varepsilon^{\mathfrak{r}} \diamond\langle\langle w-z\rangle)\right)\right)\right.\right.\right. \\
& =\varepsilon^{\mathfrak{D}} \diamond\left((\Phi A)\left\langle\left\langle\varepsilon^{A^{T}(\mathfrak{h}-\mathfrak{r})+\mathfrak{r}+\mathfrak{z}} \diamond(w-z)\right\rangle\right)\right)
\end{aligned}
$$




\section{B Auxiliary results}

Lemma 8 Assume that $s \in C^{0}([-\delta,+\infty),[0,+\infty))$, for some $\delta>0$, is such that $s(t)$ is bounded for all $t \in[-\delta, 0]$ and

$s(t) \leqslant k_{0} \int_{t-\delta}^{t} s(\tau) d \tau+k_{1}$

for all $t \geqslant 0$ and $k_{0}, k_{1} \geqslant 0$.

(i) If $\delta k_{0}<1$ then for all $t \geqslant 0$

$s(t) \leqslant \frac{k_{1}+k_{0} \delta \max _{\theta \in[-\delta, 0]} s(\theta)}{1-k_{0} \delta}$

(ii) If $k_{1}=0$ then $s(t)=0$ for all $t \geqslant \delta$.

PROOF. Part (i). We can rewrite (B.1) as

$$
s(t) \leqslant k_{0} \int_{\max \{0, t-\delta\}}^{t} s(\tau) d \tau+k_{1}^{\prime}
$$

with $k_{1}^{\prime}:=k_{1}+k_{0} \delta \max _{\theta \in[-\delta, 0]} s(\theta)$. Substituting $s(\cdot)$ in the argument of the integral with its bound in (B.3) and repeating this substitution $r$-times we obtain

$$
\begin{aligned}
& s(t) \leqslant k_{1}^{\prime} \sum_{i=0}^{r}\left(k_{0} \delta\right)^{i}+k_{0}^{r+1} \int_{\max \{0, t-\delta\}}^{t} \cdots \\
& \cdots \int_{\max \left\{0, t_{r}-\delta\right\}}^{t_{r}} s\left(t_{r+1}\right) d t_{1} \cdots d t_{r+1}
\end{aligned}
$$

By virtue of Gronwall-Bellman inequality from (B.3) it follows that $s(t) \leqslant k_{1}^{\prime} e^{k_{0} t}$. Using this inequality in (B.4) we obtain

$$
s(t) \leqslant k_{1}^{\prime} \sum_{i=0}^{r}\left(k_{0} \delta\right)^{i}+\frac{\left(k_{0} t\right)^{r+1}}{(r+1) !} k_{1}^{\prime} e^{k_{0} t}
$$

(we used the majorization $\int_{\max \{0, t-\delta\}}^{t} s^{i} e^{k_{0} s} d s \leqslant$ $\left.e^{k_{0} t} \frac{t^{i+1}}{i+1}\right)$. Passing to the limit for $r \rightarrow \infty$ and since $k_{0} \delta<1$, we get $s(t) \leqslant \frac{k_{1}^{\prime}}{1-k_{0} \delta}$ for all $t \geqslant 0$, which proves (i).

Part (ii). For $t \geqslant \delta$ we can rewrite (B.1) as $s(t) \leqslant$ $k_{0} \int_{\max \{0, t-\delta\}}^{t} s(\tau) d \tau$. Proceeding as in part (i) with $k_{1}^{\prime}:=$ 0 , we obtain $s(t)=0$ for all $t \geqslant \delta$.

Lemma 9 If $\sigma(h, \cdot) \in D^{0}\left(\mathbb{R}^{n}, \mathbb{R}^{n}\right)$ is a saturation function with levels $h \in \mathbb{R}_{>}^{n}$, for all $w, z \in \mathbb{R}^{n}$

$$
\text { (i) }\langle\langle\sigma(h, w)-\sigma(h, z)\rangle\rangle \leq 2\langle\langle w-z\rangle\rangle
$$

(ii) $\langle\langle\sigma(h, w)\rangle\rangle \leq\langle\langle w\rangle\rangle$, (iii) $\langle\langle\sigma(h, w)\rangle\rangle \leq\langle\langle h\rangle\rangle$.

PROOF. We prove only (i), while (ii) and (iii) follow easily from the definition of saturation function. Notice that $\sigma(h, w)=\left(\sigma_{1}\left(h, w_{1}\right), \ldots, \sigma_{n}\left(h, w_{n}\right)\right)^{T}$ since $\sigma(h, \cdot) \in D^{0}\left(\mathbb{R}^{n}, \mathbb{R}^{n}\right)$. Fix $i=1, \ldots, n$.

Case A). For all $w_{i}, z_{i} \in \mathbb{R}$ such that $\left|w_{i}\right| \geqslant h_{i}$ and $\left|z_{i}\right| \geqslant$ $h_{i}$ we have $\left|\sigma_{i}\left(h, w_{i}\right)-\sigma_{i}\left(h, z_{i}\right)\right|=0 \leqslant 2 \sigma_{i}\left(h,\left|w_{i}-z_{i}\right|\right) \leqslant$ $2\left|w_{i}-z_{i}\right|$ if $w_{i} z_{i}>0$ and $\left|\sigma_{i}\left(h, w_{i}\right)-\sigma_{i}\left(h, z_{i}\right)\right|=2 h_{i} \leqslant$ $2 \sigma_{i}\left(h,\left|w_{i}-z_{i}\right|\right) \leqslant 2\left|w_{i}-z_{i}\right|$ if $w_{i} z_{i}<0$.

Case B). For all $w_{i}, z_{i} \in \mathbb{R}$ such that $\left|w_{i}\right| \geqslant h_{i}$ and $\left|z_{i}\right| \leqslant h_{i}$ we have $\mid\left(\sigma_{i}\left(h, w_{i}\right)-\sigma_{i}\left(h, z_{i}\right)|=| h_{i}-z_{i} \mid=\right.$ $h_{i}-\left|w_{i}\right|+\left|w_{i}-z_{i}\right| \leqslant\left|w_{i}-z_{i}\right|=\sigma_{i}\left(h,\left|w_{i}-z_{i}\right|\right)$ if $\left|w_{i}-z_{i}\right| \leqslant h_{i}$ and $\left|\sigma_{i}\left(h, w_{i}\right)-\sigma_{i}\left(h, z_{i}\right)\right|=\left|h_{i}-z_{i}\right| \leqslant$ $2 h_{i}=2 \sigma_{i}\left(h,\left|w_{i}-z_{i}\right|\right)$ if $\left|w_{i}-z_{i}\right| \geqslant h_{i}$.

Case C). For all $w_{i}, z_{i} \in \mathbb{R}$ such that $\left|w_{i}\right| \leqslant h_{i}$ and $\left|z_{i}\right| \geqslant h_{i}$ follow the steps of case B).

In a similar way we can prove the following related result.

Lemma 10 If $\sigma(h, \cdot), \sigma(k, \cdot) \in D^{0}\left(\mathbb{R}^{n}, \mathbb{R}^{n}\right)$ are saturation functions with levels $h \in \mathbb{R}_{>}^{n}$ and, respectively, $k \in \mathbb{R}_{>}^{n},\langle\langle\sigma(h, x)-\sigma(k, x)\rangle\rangle \leq\left\langle\langle k-h\rangle\right.$ for all $x \in \mathbb{R}^{n}$.

PROOF. Fix $i=1, \ldots, n$ and assume that $h_{i} \leqslant k_{i}$ (the case $k_{i} \leqslant h_{i}$ is treated in the same way). For all $x_{i} \in \mathbb{R}$ such that $\left|x_{i}\right| \leqslant k_{i}$ we have $\left|\sigma_{i}\left(h, x_{i}\right)-\sigma_{i}\left(k, x_{i}\right)\right|=$ $0 \leqslant\left|k_{i}-h_{i}\right|$. For all other cases, we have $\mid \sigma_{i}\left(h, x_{i}\right)-$ $\sigma_{i}\left(k, x_{i}\right)|=| x_{i}-k_{i}|\leqslant| k_{i}-h_{i} \mid$.

Lemma 11 With assumption $\mathrm{HO}$ and for each $c, K>0$ and diagonal positive definite $\Gamma$,

(i) the function $(\mu, z) \mapsto \mathfrak{H}(\mu) z$, defined in (31)-(29), is i.h. with quadruple $(\mathfrak{r}, \mathfrak{r}+\mathfrak{g}, \mathfrak{g}, H)$, where $H$ is defined in (35)-(37),

(ii) the function $(\mu, z) \mapsto \mathfrak{Z}(\mu, z)$, defined in (32)-(28)(29), is i.h.u.b. with quadruple $(\mathfrak{r}, \mathfrak{r}+\mathfrak{g}, \mathfrak{g}, \Omega)$, where $\Omega$ is defined in (36)-(37) and $\Phi^{M}, \Psi^{M} \in \mathbb{R}^{n \times n}$ are matrices satisfying (20)-(21),

(iii) the functions $(\mu, z) \mapsto \lambda_{1}(\mu, z)$ and $(\mu, z) \mapsto$ $\lambda_{2}(\mu, z)$, defined in (44), are i.h.u.b. with quadruples $(\mathfrak{r}, \mathfrak{r}-\mathfrak{g}, \mathfrak{g}, 3 X)$ and, resp., $\left(\mathfrak{r}, \mathfrak{r}-\mathfrak{g}, \mathfrak{g}, C^{T}\left(C+2 \Psi^{M}\right) X\right)$, (iv) the function $z \mapsto \phi(z)$ is i.h.u.b. with quadruple $(\mathfrak{r}, \mathfrak{r}+\mathfrak{g}, \mathfrak{g}, \Phi)$,

(v) the function $(\mu, z) \mapsto A^{T} \mathfrak{G}(\mu) \mathfrak{X}(\mu) z$ is i.h.u.b. with quadruple $\left(\mathfrak{r}, \mathfrak{r}-\mathfrak{g}, \mathfrak{g}, A^{T} \Gamma X\right)$.

PROOF. Proof of part (iv). Notice that $\phi=A A^{T} \phi+$ $\left(I-A A^{T}\right) \phi$ and that $A(\mathfrak{r}-\mathfrak{g}) \leq A A^{T}(\mathfrak{r}+\mathfrak{g})$ (by (B.7) since $\left.A A^{T} A=A\right)$ and $A A^{T}(\mathfrak{r}+\mathfrak{g})+\left(I-A A^{T}\right)(\mathfrak{r}+\mathfrak{g})=$ $\mathfrak{r}+\mathfrak{g}$. From $H 0$ and $P 0, P 1$ and $P 3.1$ we get the desired result. 
Proof of parts (i), (ii) and (v). We break up the proof in several claims. Condition (5) in assumption $\mathrm{HO}$ reads out in compact form as

$$
2 A \mathfrak{g}+A A^{T}(\mathfrak{r}-\mathfrak{g}) \leq A(\mathfrak{r}-\mathfrak{g}) \leq A A^{T}(\mathfrak{r}+\mathfrak{g})
$$

and notice the following ensuing inequalities

$$
\begin{aligned}
& A^{T}(\mathfrak{r}+2 A \mathfrak{g}-\mathfrak{g}) \leq A^{T} A(\mathfrak{r}-\mathfrak{g}) \\
& A A^{T}(A \mathfrak{r}-\mathfrak{r}) \leq A A^{T}(A \mathfrak{g}+\mathfrak{g})
\end{aligned}
$$

(the first by multiplying the first inequality of (B.5) by $A^{T}$ and using $A^{T} A A^{T}=A^{T}$, the second by multiplying the second inequality of (B.5) by $A A^{T}$ and using $\left.A A^{T} A A^{T}=A A^{T}\right)$.

Claim I. $(\mu, z) \mapsto A^{T} \mathfrak{G}(\mu) z\left(\operatorname{resp} .(\mu, z) \mapsto A^{T} \mathfrak{G}^{2}(\mu) z\right)$ is i.h.u.b. with quadruple $\left(\mathfrak{r}, \mathfrak{r}-\mathfrak{g}, \mathfrak{g}, A^{T} \Gamma\right)$ (resp. $(\mathfrak{r}, \mathfrak{r}+$ $\left.\mathfrak{g}, \mathfrak{g}, A^{T} \Gamma^{2}\right)$ ). Since by its definition $(\mu, z) \mapsto \mathfrak{G}(\mu) z$ is i.h.u.b. with quadruple $(\mathfrak{r}, \mathfrak{r}, 2 A \mathfrak{g}, \Gamma)$ and $\Gamma$ is diagonal, by property $P 1$ with $\mathfrak{d}^{\prime}:=\mathfrak{r}+2 A \mathfrak{g}-\mathfrak{g}$ and $\mathfrak{h}^{\prime}:=\mathfrak{g},(\mu, z) \mapsto$ $\mathfrak{G}(\mu)$ is i.h.u.b. with quadruple $(\mathfrak{r}, \mathfrak{r}+2 A \mathfrak{g}-\mathfrak{g}, \mathfrak{g}, \Gamma)$. Вy P3.1 with $\mathfrak{z}:=\left(I-A^{T} A\right)(\mathfrak{r}-\mathfrak{g}),(\mu, z) \mapsto A^{T} \mathfrak{G}(\mu)$ is i.h.u.b. with quadruple $\left(\mathfrak{r}, A^{T}(\mathfrak{r}+2 A \mathfrak{g}-\mathfrak{g})+(I-\right.$ $\left.\left.A^{T} A\right)(\mathfrak{r}-\mathfrak{g}), \mathfrak{g}, A^{T} \Gamma\right)$. On account of (B.6) and $P 1$ we get that $(\mu, z) \mapsto A^{T} \mathfrak{G}(\mu) z$ is i.h.u.b. with quadruple $\left(\mathfrak{r}, \mathfrak{r}-\mathfrak{g}, \mathfrak{g}, A^{T} \Gamma\right)$, i.e. the first part of the claim. On the other hand, since by its definition $(\mu, z) \mapsto \mathfrak{G}^{2}(\mu) z$ is i.h.u.b. with quadruple $\left(\mathfrak{r}, \mathfrak{r}, 4 A \mathfrak{g}, \Gamma^{2}\right)$ and $\Gamma$ is diagonal, by $P 1$ with $\mathfrak{d}^{\prime}:=\mathfrak{r}+4 A \mathfrak{g}-\mathfrak{g}$ and $\mathfrak{h}^{\prime}:=\mathfrak{g},(\mu, z) \mapsto$ $\mathfrak{G}(\mu)$ is i.h.u.b. with quadruple $(\mathfrak{r}, \mathfrak{r}+4 A \mathfrak{g}-\mathfrak{g}, \mathfrak{g}, \Gamma)$. By P3.1 with $\mathfrak{z}:=\left(I-A^{T} A\right)(\mathfrak{r}+\mathfrak{g}),(\mu, z) \mapsto A^{T} \mathfrak{G}(\mu)$ is i.h.u.b. with quadruple $\left(\mathfrak{r}, A^{T}(\mathfrak{r}+4 A \mathfrak{g}-\mathfrak{g})+(I-\right.$ $\left.\left.A^{T} A\right)(\mathfrak{r}+\mathfrak{g}), \mathfrak{g}, A^{T} \Gamma^{2}\right)$. On account of (B.6) and $P 1$ we get that $(\mu, z) \mapsto A^{T} \mathfrak{G}^{2}(\mu) z$ is i.h.u.b. with quadruple $\left(\mathfrak{r}, \mathfrak{r}+\mathfrak{g}, \mathfrak{g}, A^{T} \Gamma^{2}\right)$, i.e. the second part of the claim.

Claim II. $(\mu, z) \mapsto \mathfrak{X}(\mu) z$, with $\mathfrak{X}(\mu):=\left(I-A^{T} \mathfrak{G}(\mu)\right)^{-1}$, is i.h.u.b. with quadruple $(\mathfrak{r}, \mathfrak{r}-\mathfrak{g}, \mathfrak{g}, X), \quad X \quad$ := $\left(I-A^{T} \Gamma\right)^{-1}$. Notice that $(\mu, z) \mapsto z$ is i.h.u.b. with quadruple $(\mathfrak{r}, \mathfrak{r}, 0, I)$. Therefore, since $I$ is diagonal and invoking $P 1$ with $\mathfrak{d}^{\prime}:=\mathfrak{r}+\mathfrak{g}$ and $\mathfrak{h}^{\prime}:=-\mathfrak{g},(\mu, z) \mapsto z$ is i.h.u.b. with quadruple $(\mathfrak{r}, \mathfrak{r}-\mathfrak{g}, \mathfrak{g}, I)$. On the other hand, notice that $\mathfrak{X}(\mu):=\left(I-A^{T} \mathfrak{G}(\mu)\right)^{-1}=\sum_{j=0}^{n-1}\left(A^{T} \mathfrak{G}(\mu)\right)^{j}$. As already established, $(\mu, z) \mapsto\left(A^{T} \mathfrak{G}(\mu)\right)^{0} z=z$ is i.h.u.b. with quadruple $(\mathfrak{r}, \mathfrak{r}-\mathfrak{g}, \mathfrak{g}, I)$. We proceed by induction. Assume that $(\mu, z) \mapsto\left(A^{T} \mathfrak{G}(\mu)\right)^{j} z$ for some $j=1, \ldots, n-1$, is i.h.u.b. with quadruple $(\mathfrak{r}, \mathfrak{r}-$ $\left.\mathfrak{g}, \mathfrak{g},\left(A^{T} \Gamma\right)^{j}\right)$. Since $\left(A^{T} \mathfrak{G}(\mu)\right)^{j+1}=\left(A^{T} \mathfrak{G}(\mu)\right)^{j} A^{T} \mathfrak{G}(\mu)$ and $\operatorname{both}(\mu, z) \mapsto\left(A^{T} \mathfrak{G}(\mu)\right)^{j} z$ (induction step) and $(\mu, z) \mapsto A^{T} \mathfrak{G}(\mu) z$ (claim I) are i.h.u.b. with quadruple $\left(\mathfrak{r}, \mathfrak{r}-\mathfrak{g}, \mathfrak{g},\left(A^{T} \Gamma\right)^{j}\right)$ and, respectively, $\left(\mathfrak{r}, \mathfrak{r}-\mathfrak{g}, \mathfrak{g}, A^{T} \Gamma\right)$, by property $P 2$ it follows that $(\mu, z) \mapsto\left(A^{T} \mathfrak{G}(\mu)\right)^{j+1} z$ is i.h.u.b. with quadruple $\left(\mathfrak{r}, \mathfrak{r}-\mathfrak{g}, \mathfrak{g},\left(A^{T} \Gamma\right)^{j+1}\right)$. By induction and property $P 0$, since $X:=\left(I-A^{T} \Gamma\right)^{-1}=\sum_{j=0}^{n-1}\left(A^{T} \Gamma\right)^{j}$, it follows that $(\mu, z) \mapsto \mathfrak{X}(\mu) z$ is i.h.u.b. with quadruple $(\mathfrak{r}, \mathfrak{r}-\mathfrak{g}, \mathfrak{g}, X)$. Claim III. $(\mu, z) \mapsto \mathfrak{H}(\mu) z$ is i.h. with quadruple $(\mathfrak{r}, \mathfrak{r}+\mathfrak{g}, \mathfrak{g}, H)$. Since by its definition $(\mu, z) \mapsto \mathfrak{G}(\mu) z$ is i.h. with quadruple $(\mathfrak{r}, \mathfrak{r}, 2 A \mathfrak{g}, \Gamma)$, by using property $P 3.1$ with $\mathfrak{z}:=0$ and P3.2 with $\mathfrak{z}:=\left(I-A^{T} A\right) 2 \mathfrak{g}, A^{T} G_{0} A$ is i.h. with quadruple $\left(\mathfrak{r}, A^{T} \mathfrak{r}, 2 \mathfrak{g}-A^{T} \mathfrak{r}+\mathfrak{r}, A^{T} \Gamma A\right)$. Since $A^{T} G_{0} A$ is diagonal, by $P 1$ with $\mathfrak{d}^{\prime}:=\mathfrak{r}+\mathfrak{g}$ and $\mathfrak{h}^{\prime}:=\mathfrak{g},(\mu, z) \mapsto A^{T} G_{0}(\mu) A z$ is i.h. with quadruple $\left(\mathfrak{r}, \mathfrak{r}+\mathfrak{g}, \mathfrak{g}, A^{T} \Gamma A\right)$. Similarly, $(\mu, z) \mapsto C^{T} K \mu^{2 C \mathfrak{g}} C z$ is i.h. with quadruple $\left(\mathfrak{r}, \mathfrak{r}+\mathfrak{g}, \mathfrak{g}, C^{T} K C\right)$. By $P 0$ the claim follows.

Claim IV. $(\mu, z) \mapsto \sigma\left(c \mu^{\mathfrak{r}}, z\right)$ (resp. $(\mu, z) \mapsto \sigma\left(c \mu^{\mathfrak{r}}\right.$, $\mathfrak{X}(\mu) z)$ ) are i.h.u.b. with quadruple $(\mathfrak{r}, \mathfrak{r}-\mathfrak{g}, \mathfrak{g}, 2 I)$ (resp. $(\mathfrak{r}, \mathfrak{r}-\mathfrak{g}, \mathfrak{g}, 2 X))$. On account of (i) of lemma 9 with $h:=c \mu^{\mathfrak{r}},(\mu, z) \mapsto \sigma\left(c \mu^{\mathfrak{r}}, z\right)$ is i.h.u.b. with quadruple $(\mathfrak{r}, \mathfrak{r}, 0,2 I)$. By $P 1$ with $\mathfrak{d}^{\prime}:=\mathfrak{r}-\mathfrak{g}$ and $\mathfrak{h}^{\prime}:=\mathfrak{g},(\mu, z) \mapsto$ $\sigma\left(c \mu^{\mathfrak{r}}, z\right)$ is also i.h.u.b. with quadruple $(\mathfrak{r}, \mathfrak{r}-\mathfrak{g}, \mathfrak{g}, 2 I)$, i.e. the first part of the claim. Finally, by virtue of $P 2$ and claim II we obtain the second part of the claim.

Claim V. $(\mu, z) \mapsto A \mathfrak{X}(\mu) z$ (resp. $(\mu, z) \mapsto A^{T} \mathfrak{G}(\mu)$ $\mathfrak{X}(\mu) z)$ ) is i.h.u.b. with quadruple $(\mathfrak{r}, \mathfrak{r}+\mathfrak{g}, \mathfrak{g}, A$ ) (resp. $\left.\left(\mathfrak{r}, \mathfrak{r}-\mathfrak{g}, \mathfrak{g}, A^{T} \Gamma X\right)\right)$. Note that $(\mu, z) \mapsto z$ is i.h.u.b. with quadruple $(\mathfrak{r}, \mathfrak{r}, 0, I)$, therefore by $P 1$ with $\mathfrak{d}^{\prime}:=\mathfrak{r}-\mathfrak{g}$ and $\mathfrak{h}^{\prime}:=\mathfrak{g},(\mu, z) \mapsto z$ is also i.h.u.b. with quadruple $(\mathfrak{r}, \mathfrak{r}-\mathfrak{g}, \mathfrak{g}, I)$. Using P3.1 with $\mathfrak{z}:=\left(I-A A^{T}\right)(\mathfrak{g}+\mathfrak{r})$, $(\mu, z) \mapsto A z$ is i.h.u.b. with quadruple $(\mathfrak{r}, A(\mathfrak{r}-\mathfrak{g})+(I-$ $\left.\left.A A^{T}\right)(\mathfrak{g}+\mathfrak{r}), \mathfrak{g}, A\right)$. Upon noticing that $A \mathfrak{r}-A A^{T} \mathfrak{r} \leq$ $A \mathfrak{g}+A A^{T} \mathfrak{g}$ (from (B.7) since $A A^{T} A=A$ ) and on account of (B.7), we get by $P 1$ that $(\mu, z) \mapsto A z$ is i.h.u.b. with quadruple $(\mathfrak{r}, \mathfrak{r}+\mathfrak{g}, \mathfrak{g}, A)$. From claim II and $P 2$ it follows that $(\mu, z) \mapsto A \mathfrak{X}(\mu) z$ is i.h.u.b. with quadruple $(\mathfrak{r}, \mathfrak{r}+\mathfrak{g}, \mathfrak{g}, A)$. The second part of the claim follows directly from claims I and II and $P 2$.

Claims III and V prove (i) and (v) of the lemma. Let us prove part (ii). Since $\left\|\mu^{-\mathfrak{r}} \diamond \sigma\left(c \mu^{\mathfrak{r}}, w\right)\right\| \leqslant$ cn for all $w \in \mathbb{R}^{n}$, we find out that any matrix $\Phi^{M} \in \mathbb{R}^{n \times n}$ for which (20) holds true is such that $A^{T} \Phi\left(\mu^{-\mathfrak{r}} \diamond \sigma\left(c \mu^{\mathfrak{r}}, \mathfrak{X}(\mu) w\right), \mu^{-\mathfrak{r}} \diamond \sigma\left(c \mu^{\mathfrak{r}}, \mathfrak{X}(\mu) z\right)\right) \leq$ $A^{T} \Phi^{M}$ for all $w, z \in \mathbb{R}^{n}$ and $\mu \geqslant 1$. By virtue of HO, claim IV and property $P 2$, it follows that $(\mu, z) \mapsto A^{T} \phi\left(\sigma\left(c \mu^{\mathfrak{r}}, \mathfrak{X}(\mu) z\right)\right.$ is i.h.u.b. with quadruple $\left(\mathfrak{r}, \mathfrak{r}-\mathfrak{g}, \mathfrak{g}, 2 A^{T} \Phi^{M} X\right)$. Finally, from claim III, P2 and $P 0$ and on account of part (iv) of the lemma it follows that $(\mu, z) \mapsto\left(I-\mathfrak{H}(\mu) A^{T}\right) \phi\left(\sigma\left(c \mu^{\mathfrak{r}}, \mathfrak{X}(\mu) z\right)\right.$ is i.h.u.b. with quadruple $\left(\mathfrak{r}, \mathfrak{r}+\mathfrak{g}, \mathfrak{g}, 2\left(I+H A^{T}\right) \Phi^{M} X\right)$.

In a similar way, using $H 0$, claims III, IV and $P 2$, we see that $(\mu, z) \mapsto-\mathfrak{H}(\mu) C^{T} \psi\left(\sigma\left(c \mu^{\mathfrak{r}}, \mathfrak{X}(\mu) z\right)\right)$ is i.h.u.b. with quadruple $\left(\mathfrak{r}, \mathfrak{r}+\mathfrak{g}, \mathfrak{g}, 2 H C^{T} \Psi^{M} X\right)$ for any matrix $\Psi^{M} \in \mathbb{R}^{n \times n}$ for which (21) holds true.

On the other hand, by claims I, V and $P 2$ and $P 0$, $(\mu, z) \mapsto\left[A-A^{T} \mathfrak{G}^{2}(\mu)\right] \mathfrak{X}(\mu) z$ is i.h.u.b. with quadruple $\left(\mathfrak{r}, \mathfrak{r}+\mathfrak{g}, \mathfrak{g},\left[A+A^{T} \Gamma^{2}\right] X\right)$. Using $P 0$ we obtain part (ii) of our lemma.

Proof of part (iii). As part (ii) using HO, claims II and IV and $P 0, P 2$. 


\section{References}

S. Battilotti, Incremental generalized homogeneity, observer design and semiglobal stabilization, Int. Journ. Asian Contr., 2013.

H. Smith, An introduction to delay differential equations, Texts in Applied Math., Springer Series 57, 2011.

S. Battilotti, Generalized incremental homogeneity, incremental observability and global observer design, Proc. IEEE Conf. Dec. Contr., Orlando, USA, 2011.

L.A. Marquez-Martinez, C. Moog, M. Velasco-Villa, Observability and observers with time-delay, Proc. 2nd IFAC Workshop Time Delay Systems (LTDS), Ancona (Italy), pages 52-57, 2000.

F. Mazenc, M. Malisoff, Stabilization of a chemostat model with Haldane growth functions and a delay in the measurement, IEEE Trans. on Automat. Contr., pages 1428-1436, 2010.

A. Germani, C. Manes, P. Pepe, A new approach to state observation of nonlinear systems with delayed output, IEEE Trans. on Automat. Contr., 47, pages 96-101, 2002.

S. Ibrir, Observer based control of a class of time delay nonlinear systems, Automatica, 47, pages 388-394, 2011.

J. P. Richard, Time delay systems: an overview of some recent advances and open problems, Automatica, 39, pages 388-394, 2003.

M. Krstic, Delay compensation for nonlinear adaptive and PDE systems. Birkhauser, Boston, 2009.

I. Karafyllis, M. Krstic, Predictor-based output feedback for nonlinear delay systems, Preprint, arXiv:1108.4499, 2012.

I. Karafyllis, M. Krstic, Nonlinear stabilization under sampled and delayed measurements and with inputs subject to delay and zero-order hold, IEEE Trans. Autom. Contr., 57, pages 1141-1154, 2012.

I. Karafyllis, M. Krstic, T. Ahmed-Ali, F. LamnabhiLagarrigue, Global stabilization of nonlinear delay systems with a compact absorbing set, Preprint, 2013.

I. Karafyllis, Stabilization by means of approximate predictors for systems with delayed input, SIAM Journ. Optim. Contr., 49, pages 1141-1154, 2012.

N. Kazantzis, Stabilization by means of approximate predictors for systems with delayed input, SIAM Journ. Optim. Contr., 49, pages 1141-1154, 2012.

N. Kazantzis, R. A. Wright, Nonlinear observer design in the presence of delayed output measurements, Syst. Contr. Lett., 54, pages 11001123, 2001.

N. Bekiaris-Liberis, M. Krstic, Nonlinear control under delays that depend on delayed states, Europ. Journ. Contr., 19, pages 389398, 2013.

G. Besancon, D. Georges, Z. Benayache, Asymptotic state prediction for continuous time systems with delayed input output and application to control, Proc. Europ. Contr. Conf., pages 552-557, 2007.

L. Rosier, Homogeneous Lyapunov function for homogeneous continuous vector field, Syst. Contr. Lett., 19, pages 467-473, 1992. 